\title{
Tempo de espera em centrais de atendimento com classes de clientes
}

\section{Elisa Sayuri Shimabukuro}

\author{
DISSERTAÇÃO APRESENTADA \\ $\mathrm{AO}$ \\ INSTITUTO DE MATEMÁTICA E ESTATÍSTICA \\ DA \\ UNIVERSIDADE DE SÃO PAULO \\ PARA OBTENÇÃO DO TÍTULO DE MESTRE \\ EM \\ CIÊNCIAS \\ Área de Concentração : Estatística \\ Orientador : Prof. Dr. Marcos Nascimento Magalhães \\ - São Paulo, julho de 2006 -
}




\title{
Tempo de espera em centrais de atendimento com classes de clientes
}

\author{
Este exemplar corresponde à redação final da \\ dissertação devidamente corrigida e defendida \\ por Elisa Sayuri Shimabukuro \\ e aprovada pela comissão Julgadora.
}

São Paulo, julho de 2006.

Banca examinadora:

- Prof. Dr. Marcos Nascimento Magalhães (orientador) - IME/USP

- Profa. Dra. Elizabeti Kira - IME/USP

- Profa. Dra. Graça Bressan - POLI/USP 
Aos meus pais. 


\section{Agradecimentos}

Dedico esta dissertação aos meus pais, irmãos e ao Gilson.

Agradeço ao meu orientador, Prof. Dr. Marcos Nascimento Magalhães, pelas orientações, dicas e paciência durante o mestrado.

Aos meus amigos, muito obrigada, Silvio pela ajuda na programação com o software R, Henry, Iracema, Izabel e Jacqueline pela ajuda no manuseio com o IATEX, e ao Gil pela grande ajuda na construção de todos os gráficos e desenhos deste trabalho.

Aos amigos de longa data, Célia, Gizelton, Priscila e Noemi pelo companheirismo. Enfim, a todos que de alguma fora ajudaram, torceram para que este trabalho fosse bem-sucedido, muito obrigada! 


\section{Resumo}

Este trabalho estuda o tempo médio de espera em centrais de atendimento com classes de clientes. Dois modelos para centrais são considerados: $M / M / s / r+M$ e $M / G I / s / r+G I$.

As soluções aproximadas para o tempo médio de espera, obtidas em trabalhos anteriores, foram adaptadas para o caso de várias classes de clientes.

Avaliamos numericamente as aproximações obtidas comparando-as com resultados de simulação.

Os resultados numéricos indicam uma boa qualidade na aproximação calculada. 


\section{Sumário}

1 Introdução 5

1.1 Preliminares . . . . . . . . . . . . . . . . . . . 5

1.2 Características de uma Central de Atendimento $\ldots \ldots \ldots$

1.3 Objetivo e Revisão da Literatura . . . . . . . . . . . . . . . . . . . . . 10

2 Modelo $M / M_{i} / s / r+M_{l}$ com Várias Classes 13

2.1 Introdução . . . . . . . . . . . . . . . . . . . . . 13

2.2 Tempo Médio de Espera . . . . . . . . . . . . . . . . . . . . 14

3 Tempo Médio no Modelo $M / G I_{i} / s / r+H \quad 19$

3.1 Partidas e o Tempo de Espera . . . . . . . . . . . . . . . . 19

3.2 Obtenção do Tempo Médio via Algoritmo . . . . . . . . . . . . . . . 24

3.3 Obtenção do Tempo Médio via Simulação . . . . . . . . . . . . . . . . 26

3.4 Exemplos Numéricos . . . . . . . . . . . . . . . . . 26

3.4 Parâmetros e Distribuições . . . . . . . . . . . . . . 26

3.4 .2 Análise . . . . . . . . . . . . . . . . . . . . . . 28

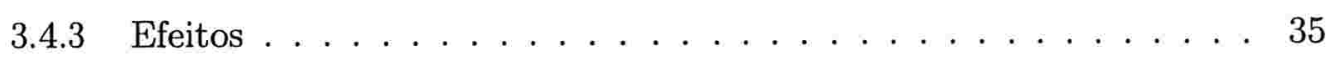

4 Conclusão $\quad 37$

4.1 Resultados Obtidos . . . . . . . . . . . . . . . . . . . . . . . 37

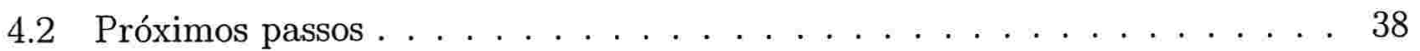


A Algoritmo e Simulação Utilizando R 40

A.1 Algoritmo . . . . . . . . . . . . . . . . . . . . 40

A.2 Simulação . . . . . . . . . . . . . . . . . . . . 44 


\section{Lista de Figuras}

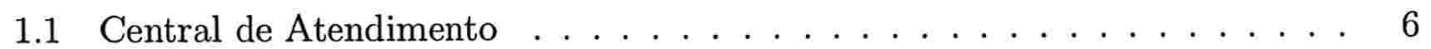

1.2 Central de Atendimento e Fila . . . . . . . . . . . . . . . 7

2.1 Tempo de Espera . . . . . . . . . . . . . . . . . . . 15

3.1 Ilustração do cálculo exato de $E W_{k}(t) \ldots \ldots \ldots \ldots \ldots \ldots$

3.2 Ilustração baseada na aproximação para $E W_{k}(t) \ldots \ldots \ldots$. . . . . . . 24

3.3 Escolha dos Parâmetros e Distribuições . . . . . . . . . . . . . . . 27

3.4 Variação no caso $k=200$ e serviços: $G_{1} \sim U(0,4)$ e $G_{2} \sim U(2,6) . \ldots . .33$

3.5 Variação no caso $k=200$ e serviços: $G_{1} \sim E(2,1)$ e $G_{2} \sim E(4,1) . \ldots . .33$

3.6 Variação no caso $k=200$ e serviços: $G_{1} \sim L N(2,1)$ e $G_{2} \sim L N(4,1)$. . . 34 


\section{Lista de Tabelas}

3.1 Distribuições de Tempo de Serviço . . . . . . . . . . . . . . . . . . 25

3.2 Tempos médios para serviços: $G_{1} \sim U(0,4)$ e $G_{2} \sim U(2,6)$. . . . . . . 29

3.3 Tempos médios para serviços: $G_{1} \sim E(2,1)$ e $G_{2} \sim E(4,1) \ldots \ldots . . .29$

3.4 Tempos médios para serviços: $G_{1} \sim L N(2,1)$ e $G_{2} \sim L N(4,1)$. . . . . 30

3.5 Tempos médios para serviços: $G_{1} \sim U(0,4)$ e $G_{2} \sim U(2,6) \ldots \ldots \ldots$

3.6 Tempos médios para serviços: $G_{1} \sim E(2,1)$ e $G_{2} \sim E(4,1)$. . . . . . 31

3.7 Tempos médios para serviços: $G_{1} \sim L N(2,1)$ e $G_{2} \sim L N(4,1) \ldots \ldots . .31$

3.8 Porcentagem dos valores da expressão aproximada contidos nos IC(95\%). . 32 


\section{Capítulo 1}

\section{Introdução}

\subsection{Preliminares}

Nos últimos anos, vem crescendo a utilização de Centrais de Atendimento em serviços públicos, vendas a varejo, bancos e canais de televisão, entre outros setores. O seu uso é divulgado através de outdoors, jornais, encartes, televisão a cabo e até mesmo em banners na Internet. É comum observarmos anúncios com os dizeres "Ligue para o nosso 0800".

Segundo o site da Associação Brasileira de Telemarketing (ABT), www.abt.org.br, o setor de Central de Atendimento emprega 580 mil pessoas atualmente no Brasil, e deve finalizar o ano de 2005 com 615 mil funcionários, a maior parte deles entre 18 e 25 anos.

Uma Central de Atendimento, também conhecida por Call Center, é uma central em que as chamadas telefônicas são processadas ou recebidas, com diversas funções, entre elas, vendas, marketing, serviço ao consumidor, telemarketing, suporte técnico, etc. O Call Center é um gerador de negócios através do telefone e um fornecedor de serviços aos clientes. Liga banco de dados, sistemas de informática, telefonia, internet e pessoas, buscando facilitar os serviços disponíveis.

Em geral, uma Central de Atendimento dispõe de tecnologia para automatizar os processos, e tem a capacidade de suportar um grande volume de ligações ao mesmo tempo, atendendo, registrando e redirecionando essas ligações quando necessário. 
$\mathrm{O}$ atendimento pode ser realizado de forma automatizada (com gravações, utilizando as chamadas "unidades de resposta por voz") ou através de atendentes ou um misto deles. A administração é complexa, dado o grande número de variáveis envolvidas em seu funcionamento, como por exemplo, previsão da quantidade de chamadas recebidas/ realizadas, contratação e gestão de pessoal, qualidade do atendimento, planejamento e gestão em tempo real. Isso explica o interesse no assunto de áreas como Pesquisa Operacional, Engenharia Industrial, Gerenciamento de Recursos Humanos, Estatística, Psicologia e Sociologia neste assunto.

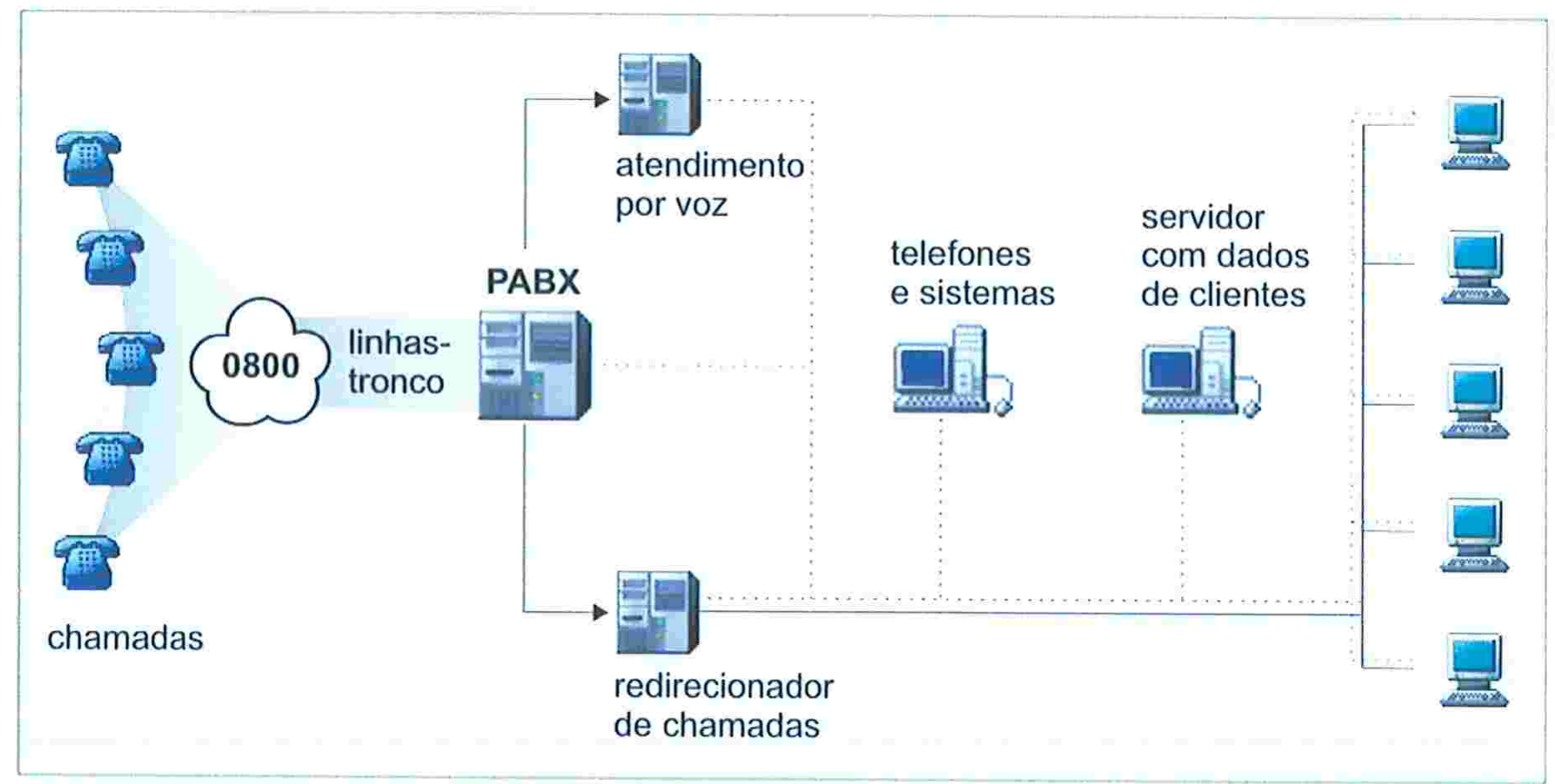

Figura 1.1: Central de Atendimento

A Figura 1.1 apresenta um diagrama esquemático exemplificando as conexões telefônicas em uma Central de Atendimento.

De acordo com recente tutorial, ver [Gans, 2005], numa situação ideal, uma central de atendimento com muitos atendentes poderia atender a milhares de chamadas telefônicas por hora, com nível médio de ocupação de atendentes entre 90\% e 95\%. Além disso, cerca de metade dos clientes receberiam atendimento quase que imediatamente, com tempo de espera medido em segundos e fração de abandono do sistema representando, no 
máximo, $2 \%$ dos clientes.

Ainda segundo o mesmo tutorial, é muito difícil encontrar uma central de atendimento com funcionamento ideal conforme as características descritas acima. A maioria das centrais de atendimento raramente apresenta ao mesmo tempo, altos níveis de eficiência e de qualidade de serviço.

Tendo em vista os recentes avanços da tecnologia e o desenvolvimento do setor de Call Center, é necessário estudar novos modelos analíticos.

Aproximações mais sofisticadas são necessárias para descrever de modo mais preciso a realidade das operações das centrais. Modelos desta realidade podem fornecer melhorias significativas na performance das centrais de atendimento.

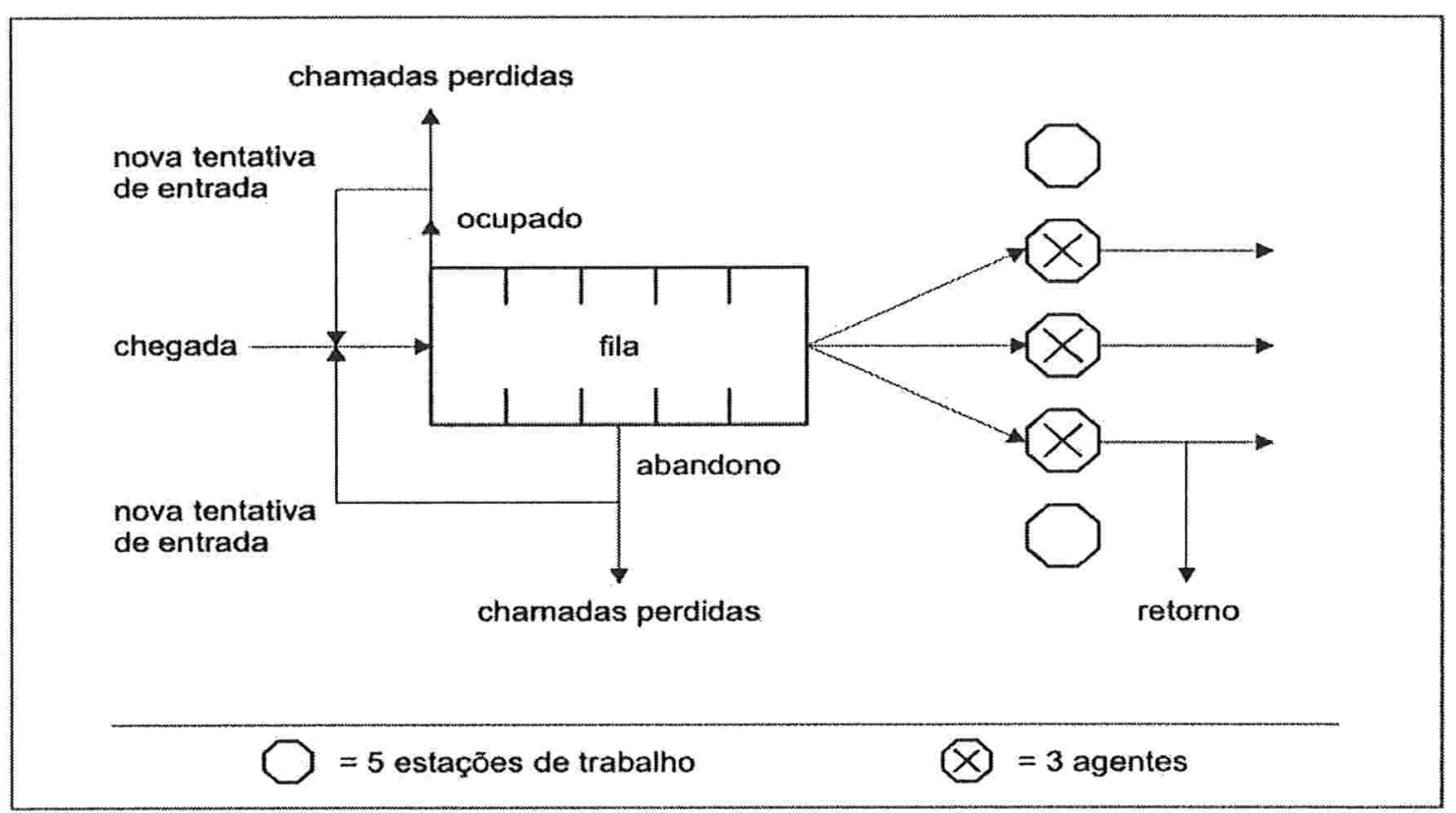

Figura 1.2: Central de Atendimento e Fila

Uma Central de Atendimento é um dos exemplos recentes de um modelo de filas. A Figura 1.2 ilustra uma central de atendimento vista como um modelo de filas.

Uma fila é formada sempre que há impossibilidade do cliente receber um serviço imediatamente. Ela pode conter clientes fisicamente, como a espera para realizar 
pagamentos em um caixa de um banco. Já uma fila virtual pode armazenar apenas a ordem de atendimento, como no caso de pacientes a espera de transplante. Mesmo que a fila exista fisicamente, o cliente pode não conseguir enxergá-la, sendo difícil avaliar a velocidade do atendimento. Uma vez na fila, um cliente poderá desistir de aguardar, de acordo com a sua paciência. O fornecimento do serviço pode ser realizado por um ou mais atendentes (servidores).

Em um fila, dependendo do cliente, o tempo de serviço pode variar bastante. Basta considerar o atendimento bancário de um office boy e de um outro cidadão qualquer. $\mathrm{O}$ office boy deve demorar mais pois leva depósitos e contas a pagar de um empresa inteira, bem diferente do caso em que o caixa atende a uma pessoa que necessita apenas efetuar o pagamento em dinheiro de uma conta de luz.

\subsection{Características de uma Central de Atendimento}

As chamadas podem ser originadas de fora da Central de Atendimento ou de dentro da própria central. O primeiro caso, contempla as centrais que prestam algum tipo de serviço como helpdesk que inclui vendas de passagens aéreas, reserva de hotéis e compras através de catálogos. Já o segundo caso, que trata de chamadas que foram originadas das centrais, englobam, de modo geral, serviços de telemarketing ou de pesquisa de mercado.

Neste trabalho focaremos nas ligações iniciadas de fora para dentro das centrais, isto é, as chamadas efetuadas pelos clientes à central.

Quando os perfis dos clientes não variam muito, as centrais não tem necessidade de possuir atendentes especializados e, portanto, cada um deles pode atender a quaisquer tipos de ligações, em geral, respeitando a ordem de chegada.

Quando a clientela é composta por pessoas com necessidades muito diferentes uma das outras temos necessidade de treinar especialistas. Neste caso, o uso de redirecionamento de chamadas é prática comum e uma chamada pode ser redirecionada para vários especialistas até que o cliente seja atendido satisfatoriamente. 
Além dos atendentes, o atendimento pode ser realizado com o auxílio de sintetizadores de voz. Isto ocorre principalmente no instante do primeiro contato do cliente com a central de atendimento.

O processo de serviço, assim como o processo de chegada das ligações, pode ser determinístico ou aleatório. Sua distribuição também pode variar de acordo com os tipos de clientes atendidos. A capacidade do sistema indica a quantidade de lugares físicos existentes para aguardar o recebimento de serviço quando todos os servidores estão ocupados. Com o barateamento das linhas telefônicas muitas centrais possuem uma ampla capacidade de linhas disponíveis.

Neste trabalho, adotaremos a seguinte convenção para descrever as características de uma fila:

$$
A / B / s / r+C,
$$

sendo,

$A$ - distribuição do tempo entre chegadas;

$B$ - distribuição do tempo de serviço;

$s$ - quantidade de servidores;

$r$ - quantidade de lugares para espera;

$C$ - distribuição do tempo que os clientes estão dispostos a esperar.

As distribuições utilizadas neste trabalho para $A, B$ e $C$ são:

- $M$ : distribuição exponencial;

- $G I$ : distribuição geral independente;

- $E$ : distribuição Erlang;

- $D$ : distribuição Determinística;

- $L N$ : distribuição Lognormal;

- $U$ : distribuição Uniforme. 
Para consultar as distribuições citadas acima, ver [Magalhães, 2006].

Quando $r$ é omitido, temos uma fila com capacidade infinita. Neste estudo, utilizaremos os modelos de filas $M / M / s / r+M$ e $M / G I / s / r+G I$.

Em uma Central de Atendimento, segundo [Feinberg e outros, 2000], o nível de serviço operacional é quantificado em termos de ocupação e medidas de perfomance. Por isso, [Feinberg e outros, 2000] sugerem o foco em medidas de performance que envolvam abandono, espera e novas tentativas de receber atendimento, através da rediscagem.

Vale ressaltar que as estatísticas do tempo médio de espera são objetivas, enquanto as medidas da paciência e de re-entrada são subjetivas pois dependem do modo como cada cliente avalia o tempo que deve esperar para ser atendido.

De modo geral, para clientes de uma Central de Atendimento, o tempo de espera é um fator crucial para determinar o sucesso do atendimento. Tempos de espera muito longos, antes do início do serviço, podem acarretar a desistência dos clientes, provocando desgastes na imagem da instituição e perda de negócios.

Sejam $S$ o evento em que o cliente entra no sistema e é atendido, e $W$ o tempo de espera que o cliente aguarda na fila.

A medida de performance $E[W \mid S]$ é a média do tempo de espera em fila dado que o cliente permaneceu em fila até ser atendido. Nesta dissertação, ela será a medida de avaliação dos modelos estudados.

\subsection{Objetivo e Revisão da Literatura}

Este trabalho tem por objetivo estudar as aproximações para o tempo de espera médio em fila nos modelos $M / M / s / r+M$ e $M / G I / s / r+G I$. Está baseado nas seções 3 e 6 do trabalho de Whitt, ver [Whitt,1999b], que considera a situação em que o cliente não abandona o sistema até receber atendimento, mesmo quando todos os servidores estão ocupados e encontra $k$ clientes na fila.

No primeiro modelo, $M / M / s / r+M$, Whitt havia desenvolvido uma aproximação para o tempo de espera médio somente para o caso em que há duas classes de clientes. 
Sendo a classe do cliente conhecida apenas do início do atendimento. Adaptamos essa aproximação para o caso em que há várias classes de clientes.

No segundo modelo, $M / G I / s / r+G I$, Whitt desenvolveu uma aproximação para o tempo médio de espera já considerando várias classes de clientes e identificando os clientes no momento em que estes se juntavam à fila. Modificamos o instante da classificação dos clientes para o instante em que estes começam a receber atendimento.

Feitas as adaptações, avaliamos as aproximações comparando-as com modelos de simulação.

Relacionados ao tema abordado neste trabalho, existem outros artigos de Whitt. Em Engineering Solution of a Basic Call Center Model, ver [Whitt, 2005], o autor apresenta uma aproximação do modelo $M / G I / s / r+G I$ através do modelo $M / M / s / r+$ $M(n)$. Aqui $M(n)$ é a distribuição da paciência que depende do estado do sistema, mais especificamente da posição do cliente na fila. Em Improving Service by informing customers about antecipated delays, ver [Whitt, 1999a], é avaliado o impacto na performance do sistema ao se fornecer, no momento da chegada de um cliente, a quantidade de usuários no sistema. Além de abandonos são considerados bloqueios e desistências no instante da chegada.

$\mathrm{O}$ artigo Asymptotic results and a Markovian approximation for the $M(n) / M(n) / s+$ GI system, ver [Brandt e outros, 2002], apresenta resultado assintótico para o modelo $M(n) / M(n) / s / r+G I$, relacionado à intensidade do processo de abandono das chamadas do sistema devido à impaciência. Eles substituíram tempos máximos de espera individuais das chamadas por taxas de impaciência dependentes da posição em que a chamada em espera ocupa na fila. O artigo [Mandelbaum, 2002] mostra o grande desafio de incorporar fatores humanos no desenvolvimento dos modelos de centrais de atendimento, oa autores sugerem que este estudo da impaciência dos clientes em fila, contemple tanto psicologia quanto marketing. Em Telephone Call Center: Tutorial, Review and Research Prospects, ver [Gans, 2005], as aproximações para os tempos de serviço e abandono, são aplicadas para centrais de atendimento que realizam redirecionamento de chamadas para clientes com perfis diferentes e, também, quando há várias classes de agentes. 
Esta dissertação está organizada na forma descrita a seguir. O Capítulo 1 apresenta uma breve introdução sobre centrais de atendimento, nomenclatura e principais problemas em estudo. O Capítulo 2 apresenta o modelo $M / M / s / r+M$ com várias classes de clientes. O Capítulo 3 trata do tempo médio em fila no modelo $M / G I / s / r+G I$ e o Capítulo 4 traz as conclusões do trabalho e possíveis pesquisas futuras. 


\section{Capítulo 2}

\section{Modelo $M / M_{i} / s / r+M_{l}$ com Várias}

\section{Classes}

\subsection{Introdução}

O interesse em considerar mais de uma classe de clientes parece natural quando, ao observarmos uma central de atendimento, percebemos que alguns clientes podem demandar mais tempo dos servidores enquanto que outros só ocupam poucos segundos. Whitt havia considerado duas classes clientes. Para o nosso modelo, consideramos a existência de $m$ classes de clientes e adotaremos a premissa, de que a classe de um cliente é identificada no momento do atendimento. Cada cliente, imediatamente antes de entrar em serviço escolhe, com probabilidade $p_{i}$, ser da classe $i$ para $i=1,2, \ldots, m$ e $\sum_{i=1}^{m} p_{i}=1$. Os tempos de serviço para cada servidor são mutuamente independentes. A distribuição do tempo de serviço, $M_{i}$, é exponencial. O cliente do tipo $i$ tem taxa de atendimento $\mu_{i}$, $i=1,2, \ldots, m$.

Além disso, o tempo de serviço para cada servidor é independente do processo de chegada.

A distribuição da paciência de um cliente, $M_{l}$, é exponencial com taxa, $\delta_{l}^{\prime}$, dependendo da sua posição $l$ na fila $\operatorname{com} l=1,2, \ldots, r$. 
Neste trabalho, não há recusa do usuário em entrar no sistema quando todos os $s$ servidores estão ocupados. Assim, todos os clientes que chegam se dispõem a ficar em fila, podendo abandoná-la mais tarde.

Para obtermos a taxa de abandono (paciência) devemos considerar que o cliente que está na fila pode abandoná-la a qualquer momento, agindo de forma independente da decisão dos outros clientes. Suponha que, o tempo que o $j$-ésimo cliente na fila está disposto a esperar na fila varia conforme uma distribuição exponencial com taxa $\delta_{j}^{\prime}$. Então, a taxa de abandono total de $k$ clientes será $\delta_{k}=\sum_{j=1}^{k} \delta_{j}^{\prime}, k \leq r$.

\subsection{Tempo Médio de Espera}

Seja $W\left(k, j_{1}, j_{2}, \ldots, j_{m}\right)$ o tempo de espera de um cliente que encontra $s+k$ clientes no sistema e que não vai abandonar a fila. Em serviço temos $j_{i}$ clientes da classe $i$, para $i=1,2, \ldots, m, \operatorname{com} \sum_{i=1}^{m} j_{i}=s$. Sempre que for conveniente, adotaremos a notação $(k, \tilde{j})$ no lugar de $\left(k, j_{1}, j_{2}, \ldots, j_{m}\right)$.

Teorema $1 O$ valor esperado de $W(k, \tilde{j})$ obedece à seguinte relação:

$$
\begin{aligned}
E[W(k, \tilde{j})] & =\frac{1}{\sum_{i=1}^{m} j_{i} \mu_{i}+\delta_{k}}+\frac{\delta_{k}}{\sum_{i=1}^{m} j_{i} \mu_{i}+\delta_{k}} E[W(k-1, \tilde{j})]+ \\
& +\frac{\sum_{d=1}^{m} \sum_{c=1}^{m} j_{d} \mu_{d} p_{c} E\left[W\left[\Delta_{c, d}(k-1, \tilde{j})\right]\right.}{\sum_{i=1}^{m} j_{i} \mu_{i}+\delta_{k}}
\end{aligned}
$$

Sendo,

$$
\Delta_{c, d}(\tilde{j})=\left(j_{1}, j_{2}, \ldots, j_{c}+1, \ldots, j_{d}-1, \ldots, j_{m}\right), \text { parac }, d=1,2, \ldots, m
$$




\section{Demonstração:}

O tempo até a primeira partida, $T_{1}$, é exponencial com média $\left(\sum_{i=1}^{s} j_{i} \mu_{i}+\delta_{k}\right)^{-1}$. Observe que a primeira partida é exponencial por ser o mínimo de várias exponenciais independentes (serviço e abandono).

Seja $T(k, \tilde{j})$ o tempo de espera restante, que começa a ser contado após a primeira partida.

O tempo de espera $W(k, \tilde{j})$ é a soma de $T_{1}$ e $T(k, \tilde{j})$ (ver Figura 2.1).

Daí, $E[W(k, \tilde{j})]=E T_{1}+E T(k, \tilde{j})$.

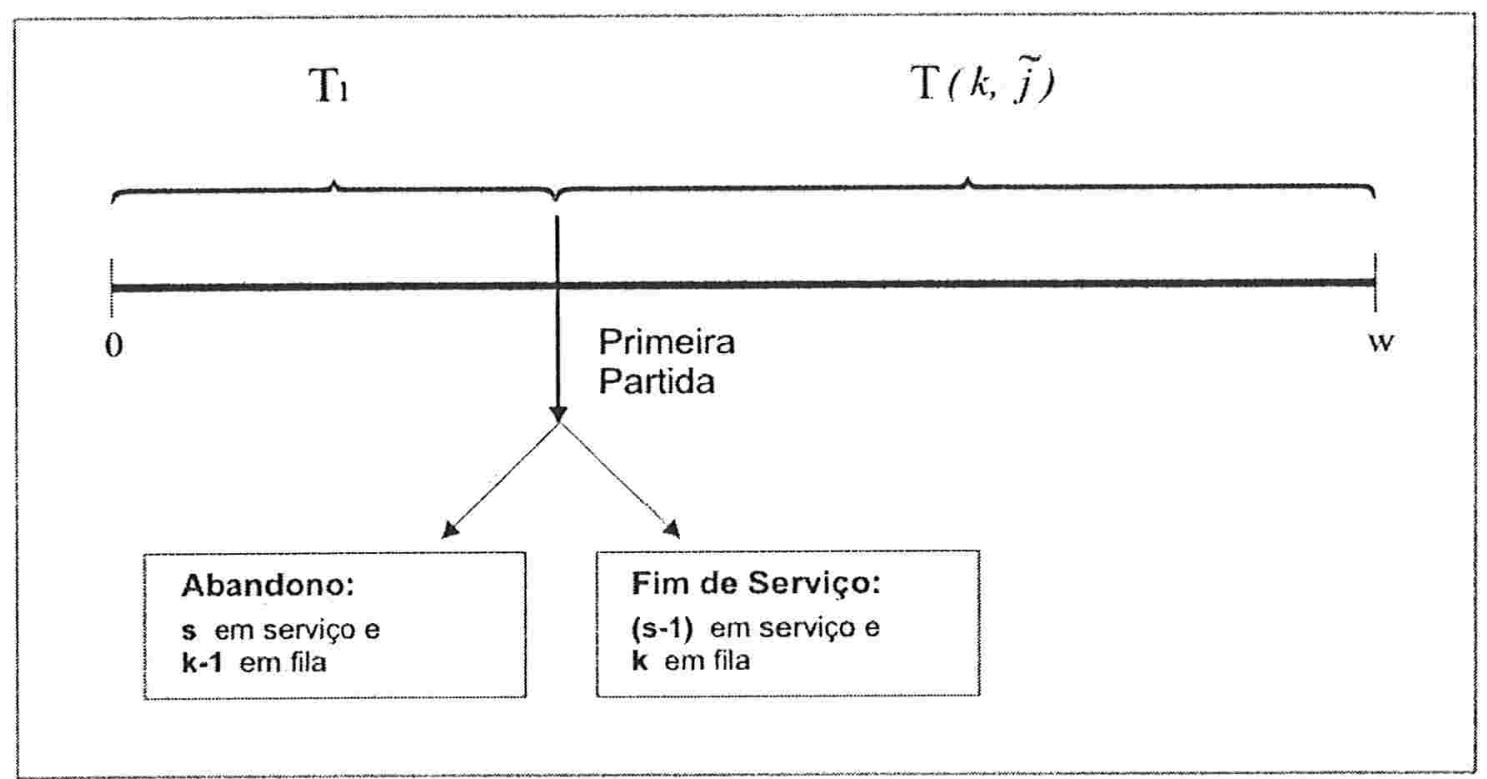

Figura 2.1: Tempo de Espera

O tempo de atendimento restante depende do evento que ocasionou a partida.

Seja $D$ a variável que caracteriza a partida.

Temos que:

$D= \begin{cases}(i, j), & \text { se um cliente da classe } i \text { completa serviço e um de classe } j \text { inicia serviço; } \\ 0, & \text { se um dos } k \text { clientes da fila desistiu. }\end{cases}$ 
Como consequiência do uso da distribuição exponencial no tempo de serviço e na paciência, e de que a classe do cliente que foi atendido não influencia na escolha da classe do que entra em serviço, vem:

$P(D=(i, j))=\frac{j_{i} \mu_{i}}{\sum_{i=1}^{m} j_{i} \mu_{i}+\delta_{k}} p_{j}$, para $i, j=1,2, \ldots, m$

$$
P(D=0) \quad=\frac{\delta_{k}}{\sum_{i=1}^{m} j_{i} \mu_{i}+\delta_{k}} .
$$

Então, para $k \geq 1$,

$$
\begin{aligned}
E[T(k, \tilde{j})] & =E[E[T(k, \tilde{j}) \mid D]] \\
& =\sum_{i=1}^{m} \sum_{j=1}^{m} E[T[(k, \tilde{j}) \mid D=(i, j)]] P[D=(i, j)] \\
& +E[T[(k, \tilde{j}) \mid D=0]] P[D=0] .
\end{aligned}
$$

No instante de uma partida, o número na fila, à frente do cliente de interesse diminui de $k$ para $k-1$. Substituindo os valores das probabilidades, temos que:

$$
\begin{aligned}
E[T(k, \tilde{j})]= & \frac{\sum_{d=1}^{m} \sum_{c=1}^{m} j_{d} \mu_{d} p_{c} E\left[W\left[\Delta_{c, d}(k-1, \tilde{j})\right]\right]}{\sum_{i=1}^{m} j_{i} \mu_{i}+\delta_{k}} \\
& +\frac{\delta_{k}}{\sum_{i=1}^{m} j_{i} \mu_{i}+\delta_{k}} E[W(k-1, \tilde{j})] .
\end{aligned}
$$

Sendo,

$$
\Delta_{c, d}(\tilde{j})=\left(j_{1}, j_{2}, \ldots,\left(j_{c}+1\right), \ldots,\left(j_{d}-1\right), \ldots, j_{m}\right), \text { para } c, d=1,2, \ldots, m
$$




$$
E[T(0, \tilde{j})]=0 .
$$

Então como

$$
E[W(k, \tilde{j})]=\frac{1}{\sum_{i=1}^{m} j_{i} \mu_{i}+\delta_{k}}+E[T(k, \tilde{j})]
$$

substituindo a expressão de $E(T(k, \tilde{j})$, temos o resultado desejado.

Desprezando a eventual dependência entre as variáveis $T_{1}$ e $T(k, \tilde{j})$, podemos obter uma aproximação para a variância do tempo.

Teorema 2 A variância tempo de espera é, aproximadamente, dada por:

$$
\begin{aligned}
\operatorname{Var}[W(k, \tilde{j})] \cong & \frac{1}{\left(\sum_{i=1}^{m} j_{i} \mu_{i}+\delta_{k}\right)}+\frac{\delta_{k}}{\sum_{i=1}^{m} j_{i} \mu_{i}+\delta_{k}} E\left[W^{2}(k-1, \tilde{j})\right] \\
& +\sum_{d=1}^{m} \sum_{c=1}^{m} j_{d} \mu_{d} p_{c}\left[\operatorname{Var}\left[W^{2}\left[\Delta_{c, d}(k-1, \tilde{j})\right]\right]+E^{2}[W(k, \tilde{j})]\right]-E^{2}[T(k, \tilde{j})] .
\end{aligned}
$$

\section{Demonstração:}

Uma recorrência equivalente à que foi desenvolvida para o valor esperado do tempo de serviço pode ser desenvolvida para a variância. Note que o tempo de espera é a soma de duas variáveis, uma é exponencial com média $\left(\sum_{i=1}^{m} j_{i} \mu_{i}+\delta_{k}\right)^{-1}$ e a outra é a variável $T(k, \tilde{j})$. Desprezando a correlação entre elas, vem

$$
\operatorname{Var}[W(k, \tilde{j})] \cong \frac{1}{\left[\sum_{i=1}^{m} j_{i} \mu_{i}+\delta_{k}\right]^{2}}+\operatorname{Var}[T(k, \tilde{j})]
$$


com

$$
\operatorname{Var}[T(k, \tilde{j})]=E\left[T^{2}(k, \tilde{j})\right]-E^{2}[T(k, \tilde{j})]
$$

como $E[T(k, \tilde{j})]$ foi calculado pelo teorema anterior, em $(2.1)$, falta calcular agora $E^{2}[T(k, \tilde{j})]$. Temos:

$$
\begin{aligned}
E\left[T^{2}(k, \tilde{j})\right]= & \frac{\sum_{d=1}^{m} \sum_{c=1}^{m} j_{d} \mu_{d} p_{c} E\left[W^{2}\left[\Delta_{c, d}(k-1, \tilde{j})\right]\right]}{\sum_{i=1}^{m} j_{i} \mu_{i}+\delta_{k}} \\
+ & \frac{\delta_{k}}{\sum_{i=1}^{m} j_{i} \mu_{i}+\delta_{k}} E\left[W^{2}(k-1, \tilde{j})\right],
\end{aligned}
$$

tal que, para $c, d=1,2, \ldots, m$,

$$
\Delta_{c, d}(\tilde{j})=\left(j_{1}, j_{2}, \ldots, j_{c}+1, \ldots, j_{d}-1, \ldots, j_{m}\right)
$$

Temos, também, que:

$$
\begin{aligned}
E\left[W^{2}(k-1, \tilde{j})\right]= & \operatorname{Var}(W(k-1, \tilde{j}))+E^{2}[W(k-1, \tilde{j})] \\
= & \frac{1}{\left(\sum_{i=1}^{m} j_{i} \mu_{i}+\delta_{k-1}\right)^{2}}+\operatorname{Var}(T(k-1, \tilde{j})) \\
& +E^{2}[W(k-1, \tilde{j})] .
\end{aligned}
$$

O resultado agora segue após as substituições correspondentes. 


\title{
Capítulo 3
}

\section{Tempo Médio no Modelo}

\author{
$M / G I_{i} / s / r+H$
}

\subsection{Partidas e o Tempo de Espera}

Este capítulo está baseado na seção 6 do artigo, ver [Whitt, 1999], que estuda o tempo de espera de um cliente que encontra $(s+k)$ clientes no sistema, e não abandona a fila até receber atendimento. Nesse artigo, a classificação dos clientes em tipos é realizada quando os clientes chegam ao sistema. Diferentemente de Whitt, manteremos a mesma premissa que adotamos no Capítulo 2, ou seja, classificamos os clientes no início do atendimento.

As aproximações para obtenção do tempo médio de espera em fila apresentadas na seção 6 do referido artigo, foram modificadas para contemplar a premissa de que a classe de um cliente é identificada no momento imediatamente antes do atendimento.

Um algoritmo é desenvolvido para calcular numericamente o valor aproximado do tempo médio de espera. E um modelo de simulação será usado para avaliar a qualidade da aproximação.

Tendo em vista que as distribuições dos tempos de serviço são não-exponenciais, para calcular o tempo médio de espera é necessário considerar os tempos já gastos pelos clientes em serviço. 
Supomos que no instante de uma nova chegada, o cliente encontra $s+k$ clientes no sistema, e são conhecidos os tempos $x_{l}, l=1,2, \ldots, s$, já gastos em serviço pelos $s$ clientes que estão sendo atendidos.

Imediatamente antes de entrar em serviço, o cliente escolhe ser do tipo $i$ com probabilidade $p_{i}, i \in\{1,2, \ldots, m\}$ e $\sum_{i=1}^{m} p_{i}=1$.

Seja $X_{i}$ o tempo de serviço de um cliente do tipo $i, i=1,2, \ldots, m$ cuja distribuição é dada por $G_{i}$.

Seja $c(l)$ a classe do cliente atendido no servidor $l, l=1,2, \ldots, s$. Supomos que esse cliente já está sendo atendido durante o tempo $x_{l}$. Então a distribuição do tempo de serviço restante condicionada ao tempo já servido é dada por:

$$
G_{c(l)}\left(t \mid x_{l}\right)=\frac{P\left[x_{l} \leq X_{c(l)} \leq x_{l}+t\right]}{P\left[X_{c(l)}>x_{l}\right]}=\frac{G_{c(l)}\left(t+x_{l}\right)-G_{c(l)}\left(x_{l}\right)}{1-G_{c(l)}\left(x_{l}\right)}, t \geq 0 .
$$

Supomos também que são conhecidos os tempos de permanência no sistema $y_{j}, j=1,2, \ldots, k$, dos $k$ clientes que estão aguardando em fila.

Considerando que alguns clientes na fila possam desistir em algum instante, seja $H_{j}\left(t \mid y_{j}\right)$ a probabilidade do cliente na $j$-ésima posição, $j=1,2 \ldots, k$, abandonar a fila, se ele não tiver sido atendido até o instante $t$.

Para simplificar a notação, considere:

$$
F_{j}\left(t \mid t_{j}, y_{j}\right)=H_{j}\left(t_{j} \mid y_{j}\right)+\left[1-H_{j}\left(t_{j} \mid y_{j}\right)\right] \sum_{i=1}^{m} p_{i} G_{i}\left(t-t_{j}\right)
$$

A quantidade $F_{j}\left(t \mid t_{j}, y_{j}\right)$ representa a probabilidade do cliente em fila na posição $j$, deixar o sistema até o instante $t$. Isto pode ser feito por abandono (primeiro termo) ou por fim de serviço (segundo termo). Nessa quantidade, $t_{j}$ é o tempo em que o cliente $j$ entraria em serviço, caso não abandonasse. 
Teorema 3 Para um cliente que não vai abandonar a fila, o tempo médio de espera obedece, de modo aproximado, à seguinte recursão:

$$
\begin{gathered}
E W_{1}(0, \tilde{j}) \cong t_{1}=\min \left\{t \geq 0: \sum_{l=1}^{s} G_{c(l)}\left(t \mid x_{l}\right)=1\right\} \quad e \\
E W_{k+1}(k, \tilde{j}) \cong t_{k+1}=\min \left\{t \geq 0: \sum_{l=1}^{s} G_{c(l)}\left(t \mid x_{l}\right)+\sum_{j=1}^{k} F_{j}\left(t \mid t_{j}, y_{j}\right)=k+1\right\}, k>0 \\
\text { tal que, } c(l) \in\{1,2, \ldots, m\} \text { e } j=1,2, \ldots, k .
\end{gathered}
$$

\section{Demonstração:}

Seja $D(t)$ o número total de partidas do sistema no intervalo $[0, t]$.

O tempo de espera é dado por:

$$
W=\min \{t \geq 0: D(t)=k+1\}
$$

$D(t)$ pode ser particionado em partidas devido ao fim de serviço (denotadas por $D_{s}(t)$ ) e partidas por desistência (denotadas por $\left.D_{k}(t)\right)$. Assim:

$$
D(t)=D_{s}(t)+D_{k}(t)
$$

Para $D_{s}(t)$ sendo o número dos $s$ clientes originais em serviço que partiram após $t$ unidades de tempo. Temos que:

$$
D_{s}(t)=\sum_{l=1}^{s} I_{s}^{l}(t),
$$

com $I_{s}^{l}$ assumindo valor 1 , se o servidor $l$ terminou de atender um cliente ou 0 , caso contrário. Assim,

$$
I_{s}^{l}(t) \sim \operatorname{Bernoulli}\left(G_{c(l)}\left(t \mid x_{l}\right)\right), t \geq 0 .
$$


Sendo $D_{k}(t)$ sendo o número dos $k$ clientes, originalmente em fila, que partiram do sistema. Temos que:

$$
D_{k}(t)=\sum_{j=1}^{k} I_{k}^{j}(t)
$$

com $I_{k}^{j}$ assumindo valor 1 se o cliente que está na posição $j, j=1,2, \ldots, k$, partiu do sistema (desistiu de aguardar em fila ou foi atendido) ou 0, caso contrário. Assim,

$$
I_{k}^{j}(t) \sim \operatorname{Bernoulli}\left(F_{j}\left(t \mid t_{j}, y_{j}\right)\right), t \geq 0 .
$$

Para calcularmos $E W$, utilizaremos a aproximação de $D(t)$ por $E D(t)$ para todo $t \geq 0$. De modo que,

$$
E W=\mathrm{E}\{\min \{t \geq 0: D(t)=k+1\}\} \cong \min \{t \geq 0: E D(t)=k+1\}
$$

. Nas Figuras 3.1 e 3.2 ilustramos um caso particular, os diferentes cálculos envolvidos nas soluções exata e aproximada de $E(W)$.

Na Figura 3.1, o cálculo seria das médias dos $t_{i}$ por mínimos, que produziria o resultado exato. Na Figura 3.2, calculamos para cada tempo fixado a média de todas as trajetórias. Do ponto de vista teórico, Whitt cita os artigos de [Whitt, 1984], e de [Berger e outros, 1992] como referência para justificar esta aproximação, mas não nos aprofundamos nessa questão.

Como:

$$
E D_{s}(t)=\sum_{l=1}^{s} G_{c(l)}\left(t \mid x_{l}\right) \quad \text { e } \quad E D_{k}(t)=\sum_{j=1}^{k} F_{j}\left(t \mid t_{j}, y_{j}\right), \quad t \geq 0
$$




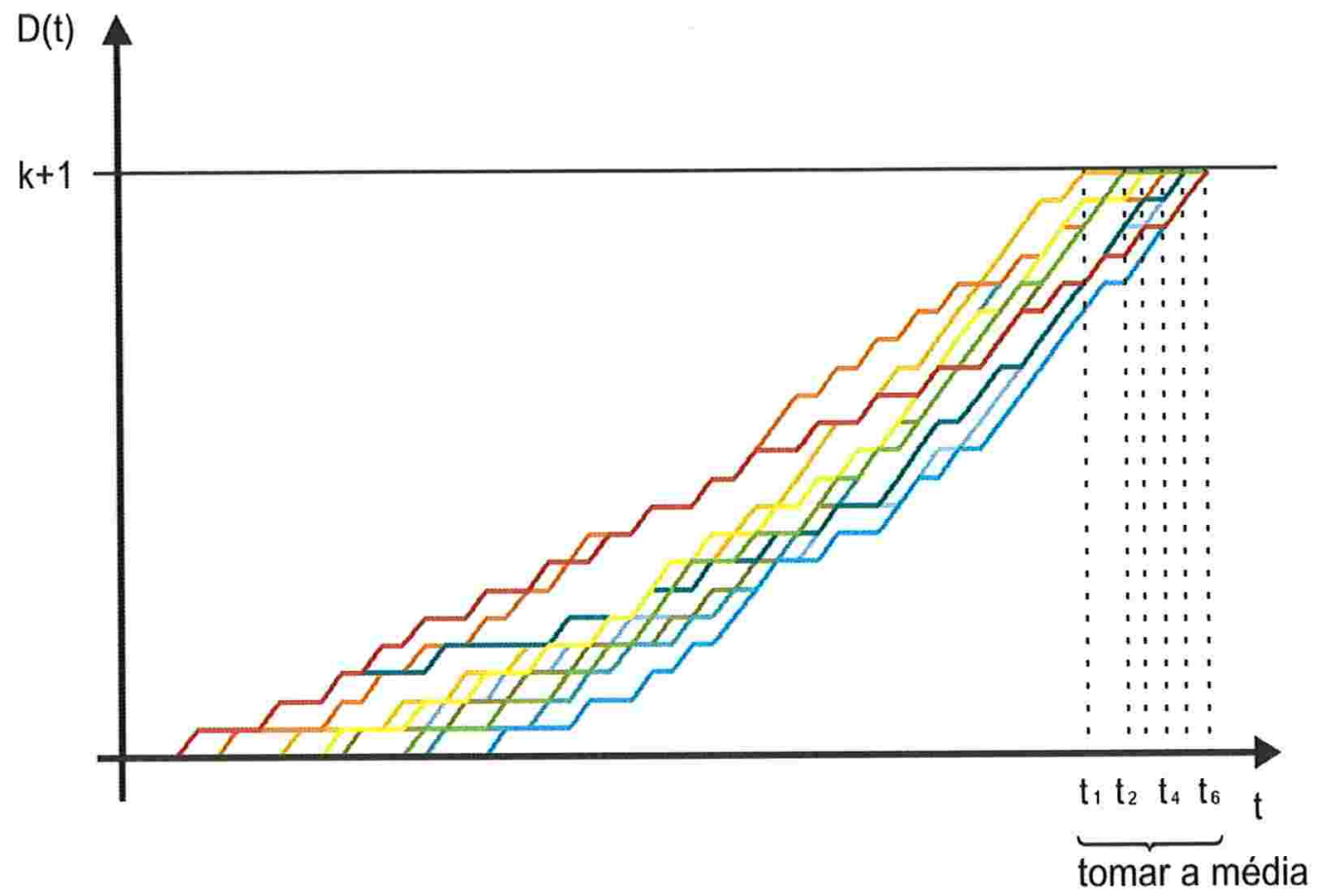

Figura 3.1: Ilustração do cálculo exato de $E W_{k}(t)$

Substituindo $E D_{s}(t)$ e $E D_{k}(t)$, segue então resultado obtido no Teorema:

$$
t_{1} \cong \min \left\{t \geq 0: \sum_{l=1}^{s} G_{c(l)}\left(t \mid x_{l}\right)=1\right\}
$$

e $t_{k+1} \cong \min \left\{t \geq 0: \sum_{l=1}^{s} G_{c(l)}\left(t \mid x_{l}\right)+\sum_{j=1}^{k} F_{j}\left(t \mid t_{j}, y_{j}\right)=k+1\right\}$ para $k>0$.

Desprezando uma eventual correlação entre as variáveis $D_{s}(t)$ e $D_{k}(t)$, podemos obter uma aproximação para a variância de $D[t]$.

Teorema 4 A variância de $D(t)$ é, aproximadamente, dada por:

$$
\operatorname{Var}[D(t)] \cong \sum_{l=1}^{s} G_{c(l)}\left(t \mid x_{l}\right)\left(1-G_{c(l)}\left(t \mid x_{l}\right)\right)+\sum_{j=1}^{k} F_{j}\left(t \mid t_{j}, y_{j}\right)\left(1-F_{j}\left(t \mid t_{j}, y_{j}\right)\right)
$$




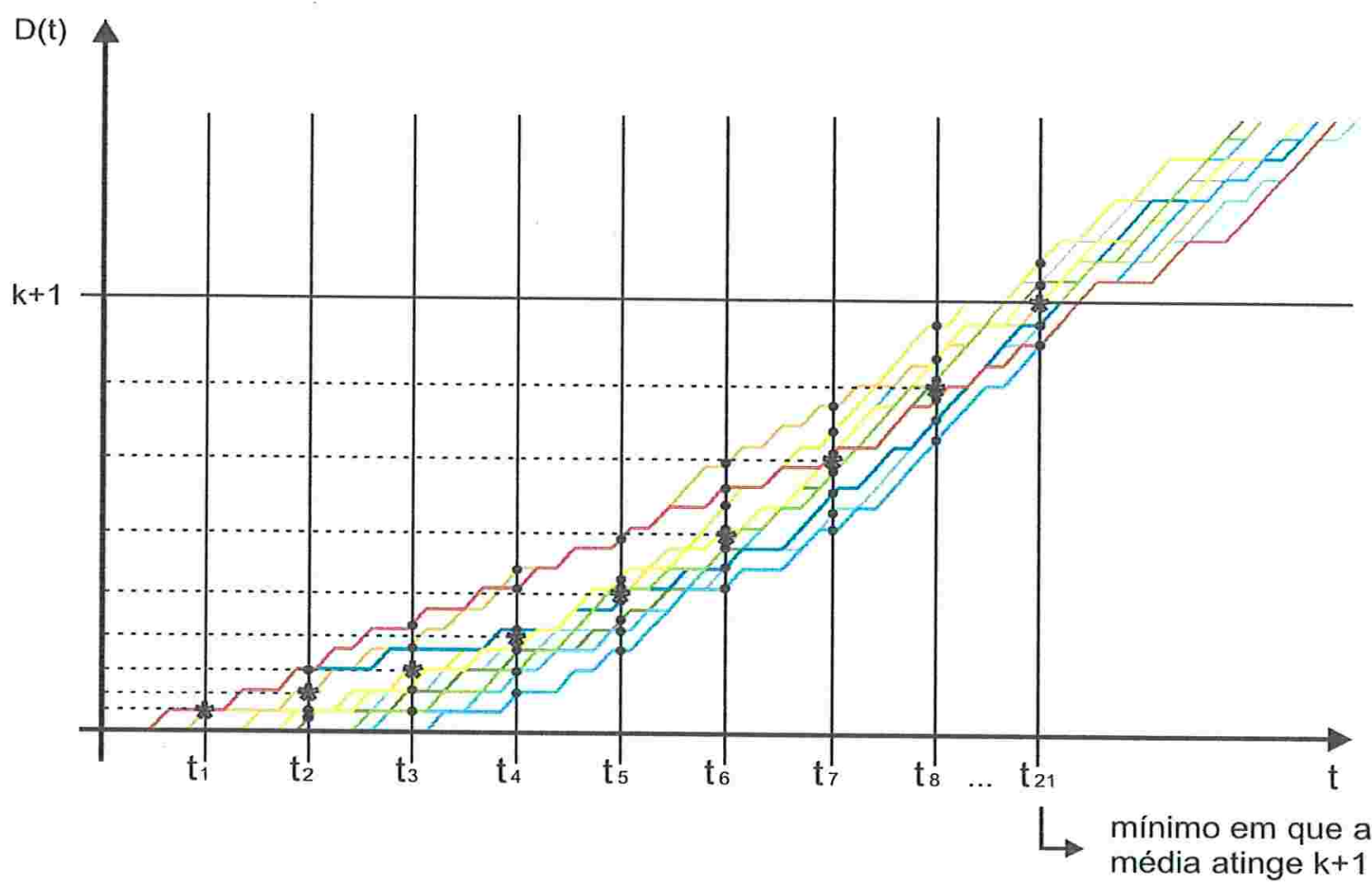

* : Valor médio considerando todas as trajetórias

Figura 3.2: Ilustração baseada na aproximação para $E W_{k}(t)$

\section{Demonstração:}

A demostração é direta, usando que $D(t)$ é soma de $D_{s}(t)$ e $D_{k}(t)$ e tomando a soma das respectivas variâncias.

\subsection{Obtenção do Tempo Médio via Algoritmo}

O algoritmo para calcular o tempo de espera médio, apresentado no Tcorema 3, foi desenvolvido no software $R$, que é uma linguagem e um ambiente para cálculos estatísticos e gráficos. É similar ao ambiente e linguagem $\mathrm{S}$ que foram desenvolvidos nos laboratórios da ATET por John Chambers e equipe. A linguagem S é utilizada no software S-Plus e é freqüentemente escolhida para pesquisa em metodologia estatística. O software $\mathrm{R}$ fornece o código aberto.

As informações acima foram retiradas do site http://www.r-project.org/, o qual traz mais detalhes sobre o software $\mathrm{R}$ e também disponibiliza uma cópia gratuita do mesmo. 
Consideramos a situação em que o cliente de número $(k+1)$ se junta a fila, e não a abandona até receber atendimento, quando encontra $(s+k)$ clientes no sistema.

Conhecemos $\tilde{x}$ e $\tilde{y}$ que são, respectivamente, o tempo de serviço já gasto em serviço pelos $s$ clientes que estão em serviço e o tempo em fila dos $k$ clientes que aguardam atendimento. Para efeito de cálculo numérico os vetores $\tilde{x}$ e $\tilde{y}$ foram escolhidos a partir de distribuições uniformes.

Enquanto o cliente aguarda em fila, não é conhecida a classe a que ele pertence, nem a dos demais clientes a sua frente. Portanto, para cada cliente que aguarda o início de seu atendimento, os tempos para abandonar a fila são independentes e identicamente distribuídos. Teremos duas opções para a distribuição da paciência.

Consideramos apenas duas classes de clientes. Os tempos de serviço são independentes, a classe de cada cliente só é escolhida no início do serviço, não antes. Foram escolhidas três opções para as distribuições dos tempos de serviço: Uniforme, Erlang e Lognormal para os tempos de serviço:

Para a distribuição da paciência, $\mathrm{H}$, representaremos clientes pouco pacientes através

\begin{tabular}{|c||c||c|}
\hline Distribuições & classe 1 & classe 2 \\
\hline & $p_{1}$ & $p_{2}=1-p_{1}$ \\
\hline Uniforme & $\mathrm{U}(0,4)$ & $\mathrm{U}(2,6)$ \\
\hline Erlang & $E(2,1)$ & $E(4,1)$ \\
\hline Lognormal & $L N(2,1)$ & $L N(4,1)$ \\
\hline
\end{tabular}

Tabela 3.1: Distribuições de Tempo de Serviço

de uma Erlang de parâmetros $(3,1)$ e média 3 pela notação $E(3,1)$ e clientes mais pacientes através de uma Erlang e média 9 pela notação $E(9,1)$.

Encontraremos os tempos médios para clientes em várias posições da fila. Em particular, para $k=1,5,10,15,50,100,200$.

Denotaremos por $\hat{t}_{k+1}$, a aproximação para o tempo médio de espera em fila do $(k+1)$-ésimo cliente, que não desiste de aguardar até que receba atendimento, obtido através deste algoritmo.

O código da programação do algoritmo pode ser consultado no Apêndice. 


\subsection{Obtenção do Tempo Médio via Simulação}

Com o uso do software $\mathrm{R}$, simulamos um modelo de filas $M / G I_{i} / s+H$, tendo as mesmas condições iniciais utilizadas no algoritmo de cálculo aproximado.

Para a simulação foram sorteados os tempos de término dos $s$ clientes que estavam em serviço, os da paciência de cada cliente na fila, o tipo do cliente que não desistiu de esperar atendimento e vai entrar em serviço e o seu tempo de serviço de acordo com a sua classe.

Obtemos o tempo médio de espera em fila do $(k+1)$-ésimo cliente que não desiste de aguardar até que receba atendimento. Foram realizadas 50 réplicas da simulação, e a média desses valores foi denotada por $\bar{t}_{k+1}$. Além disso, o desvio padrão (dp) do tempo de espera também foi calculado.

O código da programação da simulação pode ser consultado no Apêndice.

\subsection{Exemplos Numéricos}

\subsubsection{Parâmetros e Distribuições}

Apresentaremos nesta seção, os resultados numéricos obtidos com o cálculo do tempo médio via algoritmo e via simulação. Uma comparação entre eles será feita. Iniciamos descrevendo o contexto no qual faremos aplicação numérica.

As escolhas são apresentadas na Figura 3.3 e levaram em conta as distribuições teóricas freqüentes na literatura.

Por se tratar de uma central de atendimento, escolhemos grande quantidade de servidores. Assim, consideramos $s=100$.

Representaremos clientes pouco pacientes através de uma Erlang de parâmetros $(3,1)$ e média 3 pela notação $E(3,1)$ e clientes mais pacientes através de uma Erlang e média 9 pela notação $E(9,1)$. Assim, $H \sim E(3,1)$ ou $H \sim E(9,1)$

O cliente de classe 1 tem distribuição de tempo de serviço com média 2 enquanto que o cliente de classe 2 tem distribuição com média de tempo de serviço 4 . 


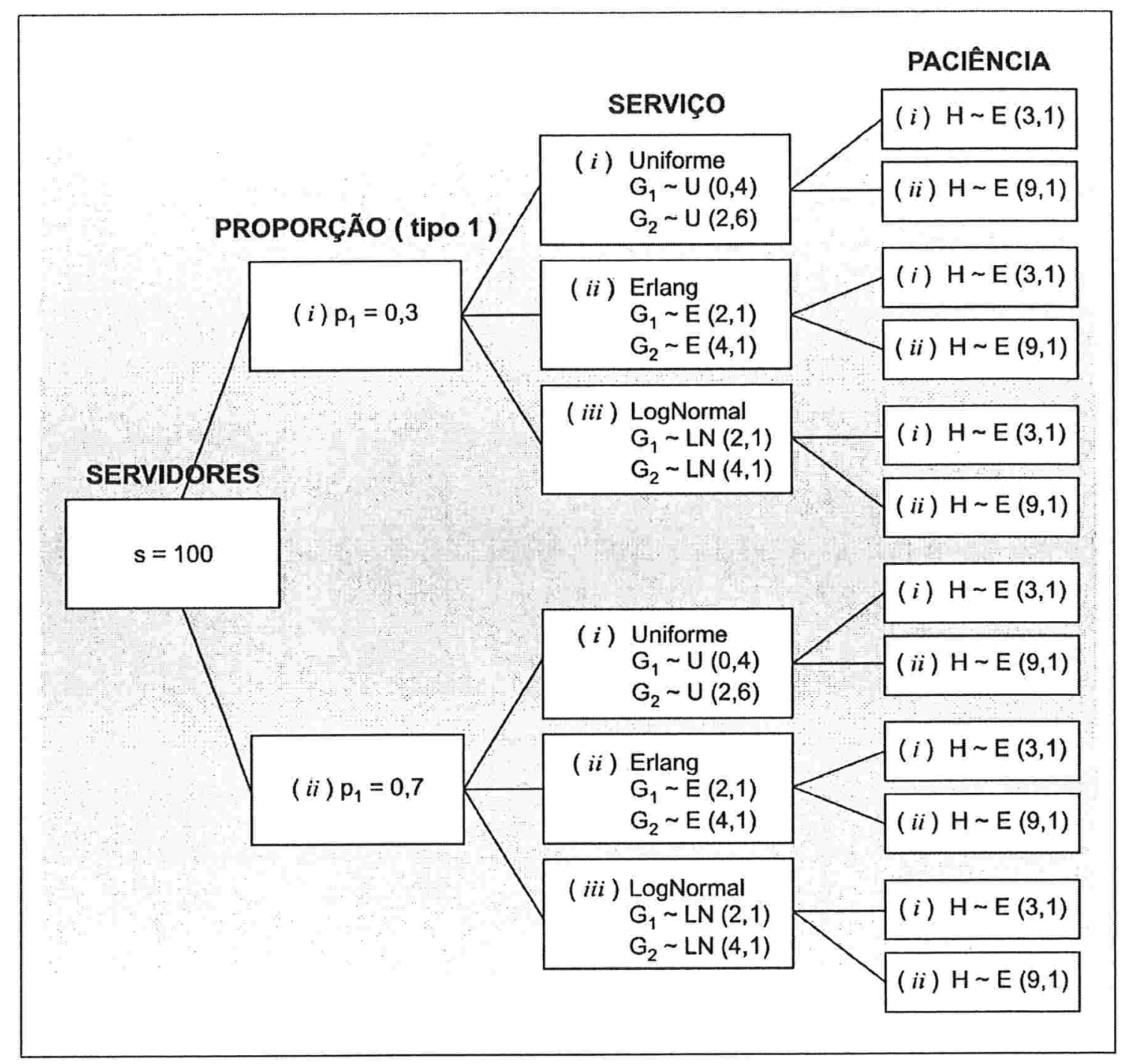

Figura 3.3: Escolha dos Parâmetros e Distribuições 
Consideramos casos em que há predominância de clientes da classe 2 com $p_{1}=0,3$ e, também em que há mais clientes da classe $1, p_{1}=0,7$.

Consideramos quatro situações para o tempo decorrido em serviço e em fila. Na primeira, denotada por $I_{1}$, os vetores $\tilde{x}$ e $\tilde{y}$ são geradas a partir de $U(0,2)$. Na segunda, denotada por $I_{2}$, esses vetores são gerados pela $U(0,3)$. A terceira, denotada por $I_{3}$, os vetores são gerados pela $U(1,75 ; 2)$. E a última, denotada por $I_{4}$, tem os vetores gerados pela $U(0 ; 0,1)$. Essas escolhas buscaram verificar se haverá ou não diferença na qualidade da aproximação em função de uma maior ou menor permanência em serviço ou em fila dos usuários presentes no sistema.

\subsubsection{Análise}

As tabelas 3.2 a 3.7 apresentam a comparação entre valores aproximado e simulado do tempo de espera médio, $E(W(k, \tilde{j})$. O valor simulado é acompanhado do desvio padrão amostral, colocado entre parenteses, que permite o cálculo do intervalo de confiança. Dessa forma, a análise será feita avaliando a inclusão ou não do valor aproximado em um intervalo de confiança de $95 \%$ obtido através de simulação. 


\begin{tabular}{|c|c|c|c|c|c|c|c|c|c|}
\hline \multicolumn{10}{|c|}{ Comparação da aproximação vs simulação } \\
\hline & & \multicolumn{4}{|c|}{$p_{1}=0,3$} & \multicolumn{4}{|c|}{$p_{1}=0,7$} \\
\hline & & \multicolumn{2}{|r|}{$I_{1}$} & \multicolumn{2}{|r|}{$I_{2}$} & \multicolumn{2}{|r|}{$I_{1}$} & \multicolumn{2}{|r|}{$I_{2}$} \\
\hline$k$ & $\mathrm{H}$ & aprox. & sim.(dp) & aprox. & sim.(dp) & aprox. & sim.(dp) & aprox. & $\operatorname{sim} .(d p)$ \\
\hline \multirow[t]{2}{*}{1} & $E(3,1)$ & 0,103 & $0,090(0,063)$ & 0,073 & $0,073(0,050)$ & 0,105 & $0,102(0,066)$ & 0,081 & $0,083(0,053)$ \\
\hline & $E(9,1)$ & 0,104 & $0,097(0,064)$ & 0,073 & $0,080(0,066)$ & 0,106 & $0,125(0,090)$ & 0,081 & $0,095(0,066)$ \\
\hline \multirow[t]{2}{*}{5} & $E(3,1)$ & $\overline{0,312}$ & $0,361(0,128)$ & 0,217 & $0,210(0,066)$ & $\overline{0,314}$ & $\overline{0,303(0,113)}$ & 0,198 & $0,182(0,074)$ \\
\hline & $E(9,1)$ & 0,320 & $0,306(0,096)$ & 0,223 & $0,212(0,089)$ & 0,322 & $0,321(0,111)$ & 0,203 & $0,205(0,076)$ \\
\hline \multirow[t]{2}{*}{10} & $\overline{E E(3,1)}$ & 0,529 & $0,494(0,152)$ & 0,390 & $0,368(0,093)$ & 0,475 & $0,449(0,118)$ & 0,374 & $0,372(0,117)$ \\
\hline & $E(9,1)$ & 0,533 & $0,514(0,147)$ & 0,402 & $0,389(0,098)$ & 0,485 & $0,490(0,131)$ & 0,385 & $0,367(0,111)$ \\
\hline \multirow[t]{2}{*}{15} & $\overline{E E(3,1)}$ & $\overline{0,646}$ & $0,649(0,133)$ & 0,486 & $0,470(0,097)$ & "0,742 & $0,748(0,169)$ & 0,498 & $0,515(0,106)$ \\
\hline & $E(9,1)$ & 0,678 & $0,671(0,144)$ & 0,518 & $0,484(0,096)$ & 0,784 & $0,821(0,168)$ & 0,531 & $0,494(0,105)$ \\
\hline \multirow[t]{2}{*}{50} & $\overline{E(3,1)}$ & 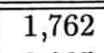 & $1,709(0,162)$ & 1,319 & $1,273(0,125)$ & 1,704 & $1,707(0,160)$ & 1,237 & $1,269(0,115)$ \\
\hline & $E(9,1)$ & 2,095 & $2,003(0,177)$ & 1,570 & $1,455(0,147)$ & 1,981 & $2,002(0,107)$ & 1,444 & $1,448(0,139)$ \\
\hline \multirow[t]{2}{*}{$\overline{100}$} & $\bar{E} E(3,1)$ & 2,518 & $2,426(0,139)$ & $\bar{~} 2,123$ & 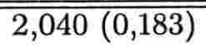 & 2,385 & 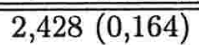 & 1,964 & $2,003(0,169)$ \\
\hline & $E(9,1)$ & 3,710 & $3,393(0,192)$ & 3,280 & $3,051(0,154)$ & 3,311 & $3,516(0,186)$ & 3,076 & $2,998(0,164)$ \\
\hline \multirow[t]{2}{*}{200} & $\begin{array}{l}E(3,1) \\
\end{array}$ & 3,477 & 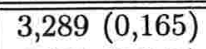 & $\overline{3,242}$ & $3,110(0,186)$ & 3,223 & $3,340(0,166)$ & $\overline{22,940}$ & $3,057(0,156)$ \\
\hline & $E(9,1)$ & 6,636 & $6,154(0,241)$ & 6,298 & $5,892(0,233)$ & 5,598 & $6,111(0,237)$ & 5,205 & $5,680(0,231)$ \\
\hline
\end{tabular}

Tabela 3.2: Tempos médios para serviços: $G_{1} \sim U(0,4)$ e $G_{2} \sim U(2,6)$.

\begin{tabular}{|c|c|c|c|c|c|c|c|c|c|}
\hline \multicolumn{10}{|c|}{ Comparação da aproximação vs simulação } \\
\hline & & \multicolumn{4}{|c|}{$p_{1}=0,3$} & \multicolumn{4}{|c|}{$p_{1}=0,7$} \\
\hline & & \multicolumn{2}{|r|}{$I_{1}$} & \multicolumn{2}{|r|}{$I_{2}$} & \multicolumn{2}{|r|}{$I_{1}$} & \multicolumn{2}{|r|}{$I_{2}$} \\
\hline$k$ & $\mathrm{H}$ & aprox. & sim.(dp) & aprox. & $\operatorname{sim} .(\mathrm{dp})$ & aprox. & sim.(dp) & aprox. & sim.(dp) \\
\hline 1 & $E(3,1)$ & 0,072 & $0,075(0,043)$ & 0,060 & $0,057(0,050)$ & 0,070 & $0,063(0,045)$ & 0,067 & $0,078(0,050)$ \\
\hline & $E(9,1)$ & 0,073 & $0,071(0,046)$ & 0,060 & $0,054(0,035)$ & 0,071 & $0,067(0,045)$ & 0,067 & $0,067(0,048)$ \\
\hline 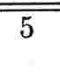 & $\begin{array}{l}E(3,1) \\
E(9,1)\end{array}$ & $\begin{array}{l}0,199 \\
0,202\end{array}$ & $\begin{array}{l}0,208(0,077) \\
0,196(0,083)\end{array}$ & $\begin{array}{l}0,180 \\
0,184\end{array}$ & $\begin{array}{l}0,182(0,074) \\
0,168(0,056)\end{array}$ & $\begin{array}{l}0,209 \\
0,213\end{array}$ & $\begin{array}{l}0,218(0,088) \\
0,203(0,084)\end{array}$ & $\begin{array}{l}0,165 \\
0,168\end{array}$ & $\begin{array}{l}0,159(0,055) \\
0,154(0,058)\end{array}$ \\
\hline 10 & $\overline{E(3,1)}$ & 0,404 & $0,399(0,107)$ & $\begin{array}{l}0,325 \\
0,334\end{array}$ & $0,301(0,078)$ & 0,404 & $\begin{array}{ll}0,399(0,098) \\
\end{array}$ & $\begin{array}{l}0,281 \\
0,287\end{array}$ & $\begin{array}{l}0,283(0,075) \\
0,299(0,078)\end{array}$ \\
\hline 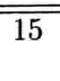 & $\begin{array}{l}E(3,1) \\
E(9,1)\end{array}$ & $\begin{array}{l}0,511 \\
0,531\end{array}$ & $\begin{array}{l}0,517(0,094) \\
0,523(0,106)\end{array}$ & $\begin{array}{l}0,428 \\
0,453\end{array}$ & $\begin{array}{l}0,414(0,087) \\
0,469(0,100)\end{array}$ & $\begin{array}{l}0,511 \\
0,531\end{array}$ & $\begin{array}{l}0,517(0,110) \\
0,526(0,112)\end{array}$ & $\begin{array}{l}0,432 \\
0,457\end{array}$ & $\begin{array}{l}0,437(0,097) \\
0,448(0,100)\end{array}$ \\
\hline 50 & $\begin{array}{l}E(3,1) \\
E(9,1)\end{array}$ & $\begin{array}{l}1,504 \\
1,752\end{array}$ & $\begin{array}{l}1,449(0,162) \\
1,660(0,171)\end{array}$ & $\begin{array}{l}1,347 \\
1,621\end{array}$ & $\begin{array}{l}1,292(0,162) \\
1,553(0,164)\end{array}$ & $\begin{array}{l}1,401 \\
1,595\end{array}$ & $\begin{array}{l}1,576(0,179) \\
1,629(0,157)\end{array}$ & $\begin{array}{l}1,227 \\
1,434\end{array}$ & $\begin{array}{l}1,433(0,161) \\
1,452(0,172)\end{array}$ \\
\hline$\overline{100}$ & $\begin{array}{l}E(3,1) \\
E(9,1)\end{array}$ & $\begin{array}{l}2,500 \\
3,614\end{array}$ & $\begin{array}{l}, 406(0,202) \\
3,348(0,220)\end{array}$ & $\begin{array}{l}2,106 \\
3,209\end{array}$ & $\begin{array}{ll}1,978 & (0,148) \\
2,934 & (0,211)\end{array}$ & $\begin{array}{l}2,257 \\
3,046\end{array}$ & $\begin{array}{l}2,642(0,204) \\
3,238(0,216)\end{array}$ & $\begin{array}{l}1,914 \\
2,713\end{array}$ & $\begin{array}{l}2,516(0,154) \\
2,920(0,224)\end{array}$ \\
\hline 200 & $\begin{array}{l}E(3,1) \\
E(9,1)\end{array}$ & $\begin{array}{l}3,433 \\
6,415\end{array}$ & $\begin{array}{l}3,273(0,174) \\
5,825(0,235)\end{array}$ & $\begin{array}{l}3,206 \\
6,230\end{array}$ & $\begin{array}{l}3,049(0,171) \\
5,737(0,250)\end{array}$ & $\begin{array}{l}3,161 \\
5,443\end{array}$ & $\begin{array}{l}3,805(0,167) \\
5,920(0,233)\end{array}$ & $\begin{array}{l}2,940 \\
5,256\end{array}$ & $\begin{array}{l}3,861(0,174) \\
5,650(0,245)\end{array}$ \\
\hline
\end{tabular}

Tabela 3.3: Tempos médios para serviços: $G_{1} \sim E(2,1)$ e $G_{2} \sim E(4,1)$. 


\begin{tabular}{|c|c|c|c|c|c|c|c|c|c|}
\hline \multicolumn{10}{|c|}{ Comparação da aproximação vs simulação } \\
\hline & & \multicolumn{4}{|c|}{$p_{1}=0,3$} & \multicolumn{4}{|c|}{$p_{1}=0,7$} \\
\hline & & \multicolumn{2}{|r|}{$I_{1}$} & \multicolumn{2}{|r|}{$I_{2}$} & \multicolumn{2}{|r|}{$I_{1}$} & \multicolumn{2}{|r|}{$I_{2}$} \\
\hline$k$ & $\mathrm{H}$ & aprox. & $\operatorname{sim} .(\mathrm{dp})$ & aprox. & $\operatorname{sim} .(d p)$ & aprox. & $\operatorname{sim} .(\mathrm{dp})$ & aprox. & $\operatorname{sim} .(\mathrm{dp})$ \\
\hline \multirow[t]{2}{*}{1} & $E(3,1)$ & 0,069 & $0,072(0,046)$ & 0,073 & $0,079(0,051)$ & 0,068 & $0,077(0,043)$ & 0,077 & $0,069(0,044)$ \\
\hline & $E(9,1)$ & 0,07 & $0,067(0,046)$ & 0,073 & $0,065(0,046)$ & 0,068 & $0,055(0,037)$ & 0,077 & $0,080(0,059)$ \\
\hline \multirow[t]{2}{*}{5} & $\overline{E(3,1)}$ & 0,21 & $0,209(0,071)$ & 0,154 & $0,157(0,060)$ & 0,267 & $0,253(0,098)$ & 0,187 & $0,173(0,068)$ \\
\hline & $E(9,1)$ & 0,213 & $0,210(0,081)$ & 0,157 & $0,157(0,054)$ & 0,272 & $0,268(0,099)$ & 0,191 & $0,171(0,065)$ \\
\hline \multirow[t]{2}{*}{10} & $\overline{E(3,1)}$ & 0,443 & $0,419(0,120)$ & 0,306 & $0,321(0,084)$ & 0,420 & $0,455(0,101)$ & 0,266 & $0,253(0,076)$ \\
\hline & $E(9,1)$ & 0,452 & $0,458(0,101)$ & 0,314 & $0,300(0,064)$ & 0,428 & $0,440(0,095)$ & 0,272 & $0,268(0,082)$ \\
\hline \multirow[t]{2}{*}{15} & $\overline{E(3,1)}$ & 0,57 & $0,578(0,092)$ & 0,451 & $0,434(0,094)$ & 0,629 & $0,677(0,131)$ & 0,431 & $0,430(0,106)$ \\
\hline & $E(9,1)$ & 0,594 & $0,566(0,095)$ & 0,479 & $0,473(0,099)$ & 0,66 & $0,652(0,129)$ & 0,456 & $0,453(0,117)$ \\
\hline \multirow[t]{2}{*}{ "50 } & $\overline{E(3,1)}$ & 1,694 & $1,848(0,131)$ & 1,266 & $\bar{~} 1,348(0,110)$ & 1,481 & $1,662(0,144)$ & 1,311 & $1,391(0,132)$ \\
\hline & $E(9,1)$ & 2,015 & $1,938(0,118)$ & 1,503 & $1,477(0,136)$ & 1,708 & $1,740(0,127)$ & 1,543 & $1,568(0,119)$ \\
\hline \multirow[t]{2}{*}{100} & $\overline{E(3,1)}$ & 2,51 & $2,577(0,118)$ & 2,103 & $2,051(0,120)$ & 2,32 & $2,556(0,136)$ & 1,989 & $2,081(0,135)$ \\
\hline & $E(9,1)$ & 3,601 & $3,331(0,143)$ & 3,237 & $2,947(0,170)$ & 3,123 & $3,305(0,169)$ & 2,821 & $3,002(0,168)$ \\
\hline \multirow[t]{2}{*}{200} & $\overline{E(3,1)}$ & 3,465 & $3,266(0,123)$ & 3,225 & $2,915(0,139)$ & 3,262 & $3,311(0,111)$ & 2,914 & $2,897(0,137)$ \\
\hline & $E(9,1)$ & 6,59 & $6,306(0,198)$ & 6,209 & $5,948(0,159)$ & 5,708 & $6,484(0,202)$ & 5,222 & $5,942(0,184)$ \\
\hline
\end{tabular}

Tabela 3.4: Tempos médios para serviços: $G_{1} \sim L N(2,1)$ e $G_{2} \sim L N(4,1)$.

\begin{tabular}{|c|c|c|c|c|c|c|c|c|c|}
\hline \multicolumn{10}{|c|}{ Comparação da aproximação vs simulação } \\
\hline & & \multicolumn{4}{|c|}{$p_{1}=0,3$} & \multicolumn{4}{|c|}{$p_{1}=0,7$} \\
\hline & & \multicolumn{2}{|r|}{$I_{3}$} & \multicolumn{2}{|r|}{$I_{4}$} & \multicolumn{2}{|r|}{$I_{3}$} & \multicolumn{2}{|r|}{$I_{4}$} \\
\hline$k$ & $\mathrm{H}$ & aprox. & $\operatorname{sim} .(\mathrm{dp})$ & aprox. & $\operatorname{sim} .(\mathrm{dp})$ & aprox. & $\operatorname{sim} .(\mathrm{dp})$ & aprox. & $\operatorname{sim} .(\mathrm{dp})$ \\
\hline \multirow[t]{2}{*}{$\frac{\omega}{1}$} & $E(3,1)$ & 0,042 & $0,041(0,024)$ & 0,135 & $0,115(0,069)$ & 0,003 & $0,002(0,002)$ & 0,167 & $0,153(0,097)$ \\
\hline & $E(9,1)$ & 0,042 & $0,036(0,025)$ & 0,135 & $0,141(0,106)$ & 0,003 & $0,002(0,002)$ & 0,167 & $0,132(0,080)$ \\
\hline \multirow[t]{2}{*}{ 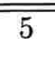 } & $E(3,1)$ & 0,204 & $0,209(0,079)$ & 0,516 & $0,542(0,207)$ & 0,202 & $\overline{00,203(0,061)}$ & 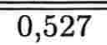 & 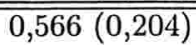 \\
\hline & $E(9,1)$ & 0,210 & $0,204(0,082)$ & 0,518 & $0,557(0,179)$ & 0,208 & $0,218(0,068)$ & 0,530 & $0,503(0,187)$ \\
\hline \multirow[t]{2}{*}{$\overline{10}$} & $\overline{E(3,1)}$ & 0,230 & $0,251(0,070)$ & 0,760 & $0,722(0,200)$ & 0,032 & $0,032(0,008)$ & 0,834 & $\overline{0,860(0,206)}$ \\
\hline & $E(9,1)$ & 0,232 & $0,227(0,070)$ & 0,769 & $0,715(0,187)$ & 0,032 & $0,033(0,009)$ & 0,846 & $0,869(0,214)$ \\
\hline \multirow[t]{2}{*}{15} & $E(3,1)$ & 0,329 & $0,302(0,062)$ & 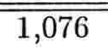 & $\overline{1} 1,027(0,226)$ & 0,053 & $0,050(0,013)$ & 0,967 & $\overline{1,015(0,219)}$ \\
\hline & $E(9,1)$ & 0,334 & $0,307(0,076)$ & 1,107 & $1,032(0,260)$ & 0,053 & $0,053(0,015)$ & 0,988 & $0,976(0,207)$ \\
\hline \multirow[t]{2}{*}{50} & $E(3,1)$ & 0,960 & $0,953(0,102)$ & 2,424 & $2,358(0,151)$ & 0,686 & $0,718(0,127)$ & 2,292 & $2,328(0,168)$ \\
\hline & $E(9,1)$ & 1,045 & $1,010(0,118)$ & 2,820 & $2,691(0,162)$ & 0,716 & $0,760(0,124)$ & 2,605 & $2,663(0,173)$ \\
\hline \multirow[t]{2}{*}{100} & $E(3,1)$ & 1,647 & $1,595(0,094)$ & 3,128 & $3,061(0,174)$ & 1,609 & $1,717(0,152)$ & 3,054 & $3,085(0,157)$ \\
\hline & $E(9,1)$ & 1,992 & $1,892(0,093)$ & 4,286 & $3,988(0,218)$ & 1,862 & $2,037(0,150)$ & 3,966 & $4,172(0,243)$ \\
\hline \multirow[t]{2}{*}{200} & $E(3,1)$ & 3,266 & $2,956(0,248)$ & 4,085 & $3,970(0,194)$ & 2,866 & $3,045(0,150)$ & 3,771 & $3,3,876(0,147)$ \\
\hline & $E(9,1)$ & 5,745 & $5,128(0,243)$ & 7,346 & $6,788(0,298)$ & 4,604 & $5,161(0,216)$ & 6,138 & $6,595(0,207)$ \\
\hline
\end{tabular}

Tabela 3.5: Tempos médios para serviços: $G_{1} \sim U(0,4)$ e $G_{2} \sim U(2,6)$. 


\begin{tabular}{|c|c|c|c|c|c|c|c|c|c|}
\hline \multicolumn{10}{|c|}{ Comparação da aproximação vs simulação } \\
\hline & & \multicolumn{4}{|c|}{$p_{1}=0,3$} & \multicolumn{4}{|c|}{$p_{1}=0,7$} \\
\hline & & \multicolumn{2}{|r|}{$I_{3}$} & \multicolumn{2}{|r|}{$I_{4}$} & \multicolumn{2}{|r|}{$I_{3}$} & \multicolumn{2}{|r|}{$I_{4}$} \\
\hline$k$ & $\mathrm{H}$ & aprox. & $\operatorname{sim} .(\mathrm{dp})$ & aprox. & sim.(dp) & aprox. & $\operatorname{sim} .(\mathrm{dp})$ & aprox. & $\operatorname{sim} .(\mathrm{dp})$ \\
\hline \multirow[t]{2}{*}{1} & $E(3,1)$ & 0,038 & $0,043(0,028)$ & 0,260 & $0,229(0,117)$ & 0,039 & $0,041(0,029)$ & 0,258 & $0,23(0,094)$ \\
\hline & $E(9,1)$ & 0,038 & $0,037(0,037)$ & 0,260 & $0,220(0,096)$ & 0,039 & $0,041(0,028)$ & 0,258 & $0,236(0,097)$ \\
\hline \multirow[t]{2}{*}{5} & $E(3,1)$ & 0,137 & $0,139(0,054)$ & 0,545 & $0,52(0,145)$ & 0,142 & $0,141(0,060)$ & 0,624 & $0,587(0,145)$ \\
\hline & $E(9,1)$ & 0,140 & $0,144(0,056)$ & 0,547 & $0,558(0,125)$ & 0,146 & $0,152(0,055)$ & 0,627 & $0,600(0,133)$ \\
\hline \multirow[t]{2}{*}{10} & $E(3,1)$ & 0,219 & $0,22(0,065)$ & 0,817 & $0,796(0,139)$ & 0,226 & $0,225(0,075)$ & 0,773 & $0,739(0,134)$ \\
\hline & $E(9,1)$ & 0,221 & $0,210(0,049)$ & 0,828 & $0,795(0,149)$ & 0,228 & $0,216(0,060)$ & 0,782 & $0,769(0,130)$ \\
\hline \multirow[t]{2}{*}{15} & $\overline{E(3,1)}$ & 0,307 & $0,313(0,079)$ & 1,004 & $0,972(0,132)$ & 0,326 & $0,338(0,069)$ & 0,948 & $0,907(0,151)$ \\
\hline & $E(9,1)$ & 0,312 & $0,308(0,077)$ & 1,027 & $0,993(0,172)$ & 0,331 & $0,313(0,081)$ & 0,967 & $0,968(0,150)$ \\
\hline \multirow[t]{2}{*}{50} & $E(3,1)$ & 1,082 & $1,036(0,102)$ & 2,101 & $1,994(0,175)$ & 1,036 & $1,125(0,155)$ & 1,910 & $1,968(0,159)$ \\
\hline & $E(9,1)$ & 1,209 & $1,184(0,130)$ & 2,413 & $2,301(0,174)$ & 1,144 & $1,173(0,100)$ & 2,130 & $2,182(0,183)$ \\
\hline \multirow[t]{2}{*}{100} & $E(3,1)$ & 2,028 & $1,917(0,139)$ & 2,965 & $2,832(0,143)$ & 1,878 & $2,275(0,177)$ & 2,911 & $3,000(0,154)$ \\
\hline & $E(9,1)$ & 2,745 & $2,511(0,202)$ & 4,029 & $3,805(0,234)$ & 2,408 & $2,562(0,180)$ & 3,809 & $3,971(0,287)$ \\
\hline \multirow[t]{2}{*}{200} & $\overline{E(3,1)}$ & $\overline{3,220}$ & $2,993(0,184)$ & 4,014 & $3,836(0,215)$ & 2,92 & $3,644(0,182)$ & 3,768 & $3,901(0,165)$ \\
\hline & $E(9,1)$ & 5,960 & $5,454(0,212)$ & 6,978 & $6,458(0,308)$ & 4,97 & $5,402(0,244)$ & 6,076 & $6,504(0,237)$ \\
\hline
\end{tabular}

Tabela 3.6: Tempos médios para serviços: $G_{1} \sim E(2,1)$ e $G_{2} \sim E(4,1)$.

\begin{tabular}{|c|c|c|c|c|c|c|c|c|c|}
\hline \multicolumn{10}{|c|}{ Comparação da aproximação vs simulação } \\
\hline & & \multicolumn{4}{|c|}{$p_{1}=0,3$} & \multicolumn{4}{|c|}{$p_{1}=0,7$} \\
\hline & & \multicolumn{2}{|r|}{$I_{3}$} & \multicolumn{2}{|r|}{$I_{4}$} & \multicolumn{2}{|r|}{$I_{3}$} & \multicolumn{2}{|r|}{$I_{4}$} \\
\hline$k$ & $\mathrm{H}$ & aprox. & sim.(dp) & aprox. & sim.(dp) & aprox. & $\operatorname{sim} .(\mathrm{dp})$ & aprox. & sim.(dp) \\
\hline \multirow[t]{2}{*}{1} & $E(3,1)$ & 0,024 & $0,024(0,014)$ & 0,729 & $0,729(0,119)$ & 0,028 & $0,034(0,025)$ & 0,726 & $0,690(0,110)$ \\
\hline & $E(9,1)$ & 0,024 & $0,025(0,017)$ & 0,732 & $0,714(0,115)$ & 0,028 & $0,027(0,023)$ & 0,729 & $0,690(0,122)$ \\
\hline \multirow[t]{2}{*}{5} & $E(3,1)$ & 0,117 & $0,108(0,038)$ & 0,974 & $0,961(0,110)$ & $\overline{0,134}$ & $0,137(0,051)$ & 0,976 & $0,966(0,104)$ \\
\hline & $E(9,1)$ & 0,119 & $0,116(0,046)$ & 0,987 & $0,972(0,103)$ & 0,137 & $0,121(0,043)$ & 0,989 & $0,980(0,115)$ \\
\hline \multirow[t]{2}{*}{10} & $\overline{E(3,1)}$ & 0,157 & ( & $\overline{11,139}$ & $1,164(0,138)$ & 0,153 & $0,152(0,047)$ & 1,131 & $1,135(0,107)$ \\
\hline & $E(9,1)$ & 0,158 & $0,159(0,041)$ & 1,167 & $1,156(0,103)$ & 0,153 & $0,142(0,044)$ & 1,157 & $1,173(0,112)$ \\
\hline \multirow[t]{2}{*}{15} & $E(3,1)$ & 0,226 & $0,222(0,064)$ & 1,362 & $1,410(0,128)$ & 0,259 & $0,259(0,068)$ & 1,389 & $1,409(0,131)$ \\
\hline & $E(9,1)$ & 0,228 & $0,224(0,054)$ & 1,421 & $1,440(0,142)$ & 0,262 & $0,240(0,059)$ & 1,454 & $1,419(0,144)$ \\
\hline \multirow[t]{2}{*}{50} & $\overline{E(3,1)}$ & 0,827 & $0,887(0,111)$ & 2,257 & $2,442(0,133)$ & $\overline{c 0,966}$ & $1,055(0,098)$ & 2,518 & $\overline{2,722(0,123)}$ \\
\hline & $E(9,1)$ & 0,896 & $0,892(0,110)$ & 2,709 & $2,620(0,136)$ & 1,059 & $1,036(0,116)$ & 2,966 & $3,025(0,141)$ \\
\hline \multirow[t]{2}{*}{100} & $\overline{E E(3,1)}$ & 1,890 & $2,074(0,131)$ & 3,268 & $3,290(0,128)$ & 1,744 & $2,077(0,134)$ & 3,147 & $3,293(0,105)$ \\
\hline & $E(9,1)$ & 2,553 & $2,312(0,165)$ & 4,462 & $4,221(0,150)$ & 2,158 & $2,275(0,150)$ & 4,091 & $4,262(0,143)$ \\
\hline \multirow[t]{2}{*}{200} & $\overline{E(3,1)}$ & 3,320 & $3,091(0,145)$ & 4,078 & $3,876(0,109)$ & 5,807 & $5,412(0,202)$ & 7,286 & $7,136(0,235)$ \\
\hline & $E(9,1)$ & 5,807 & $5,412(0,202)$ & 7,286 & $7,136(0,235)$ & 5,807 & $5,412(0,202)$ & 7,286 & $7,136(0,235)$ \\
\hline
\end{tabular}

Tabela 3.7: Tempos médios para serviços: $G_{1} \sim L N(2,1)$ e $G_{2} \sim L N(4,1)$. 
Para avaliarmos em que contexto os valores da aproximação são razoáveis, fixando as condições iniciais e a quantidade de clientes à frente na fila, temos um total de 12 valores (combinando 2 proporções de clientes do tipo 1, 3 distribuições de tempos de serviço e 2 distribuições de paciência) a serem analisados.

Cada porcentagem apresentada na Tabela 3.8 representa a quantidade de valores, dos 12 já mencionados, em que o valor aproximado está contido no intervalo de confiança $(\gamma=95 \%)$ para o valor simulado, de acordo com o respectivo valor do desvio padrão amostral apresentando nas Tabelas 3.3 a 3.7 .

\begin{tabular}{|c||c||c||c|}
\hline \multicolumn{4}{|c|}{ Tempo médio de espera } \\
\hline Condiçôes Iniciais & $k<100$ & $k=100$ & $k=200$ \\
\hline$I_{1}$ & $100 \%$ & $100 \%$ & $50 \%$ \\
\hline$I_{2}$ & $100 \%$ & $92 \%$ & $58 \%$ \\
\hline$I_{3}$ & $100 \%$ & $83 \%$ & $42 \%$ \\
\hline$I_{4}$ & $100 \%$ & $100 \%$ & $83 \%$ \\
\hline
\end{tabular}

Tabela 3.8: Porcentagem dos valores da expressão aproximada contidos nos IC(95\%).

Para obter uma melhor visualização dos dados apresentados nas Tabelas 3.2 a 3.7, apresentaremos gráficos que mostram quão perto estão os valores dos tempos aproximados e simulados. A medida que utilizamos para mensurar esta proximidade será denominada por variação, e sua fórmula é dada por:

$$
\text { variação }=\left[\frac{\text { aproximação }- \text { simulação }}{\text { simulação }}\right] * 100 .
$$

Notamos que, em geral, na Tabela 3.8, para $k=200$, essa porcentagem é baixa, por volta dos $50 \%$. A exceção foi a condição inicial que representa a situação em que os clientes estão há pouco tempo no sistema, com $84 \%$. Como a diferença entre o tempo de espera médio obtido através da aproximação e da simulação não foi significativa nas posições $k$ de 1 a 100 (ver Tabela 3.8), foram construídos alguns gráficos de variação, somente para a posição $k=200$, considerando as quatro condições iniciais utilizadas.

Observando os gráficos acima, verificamos que o valor da aproximação não está 


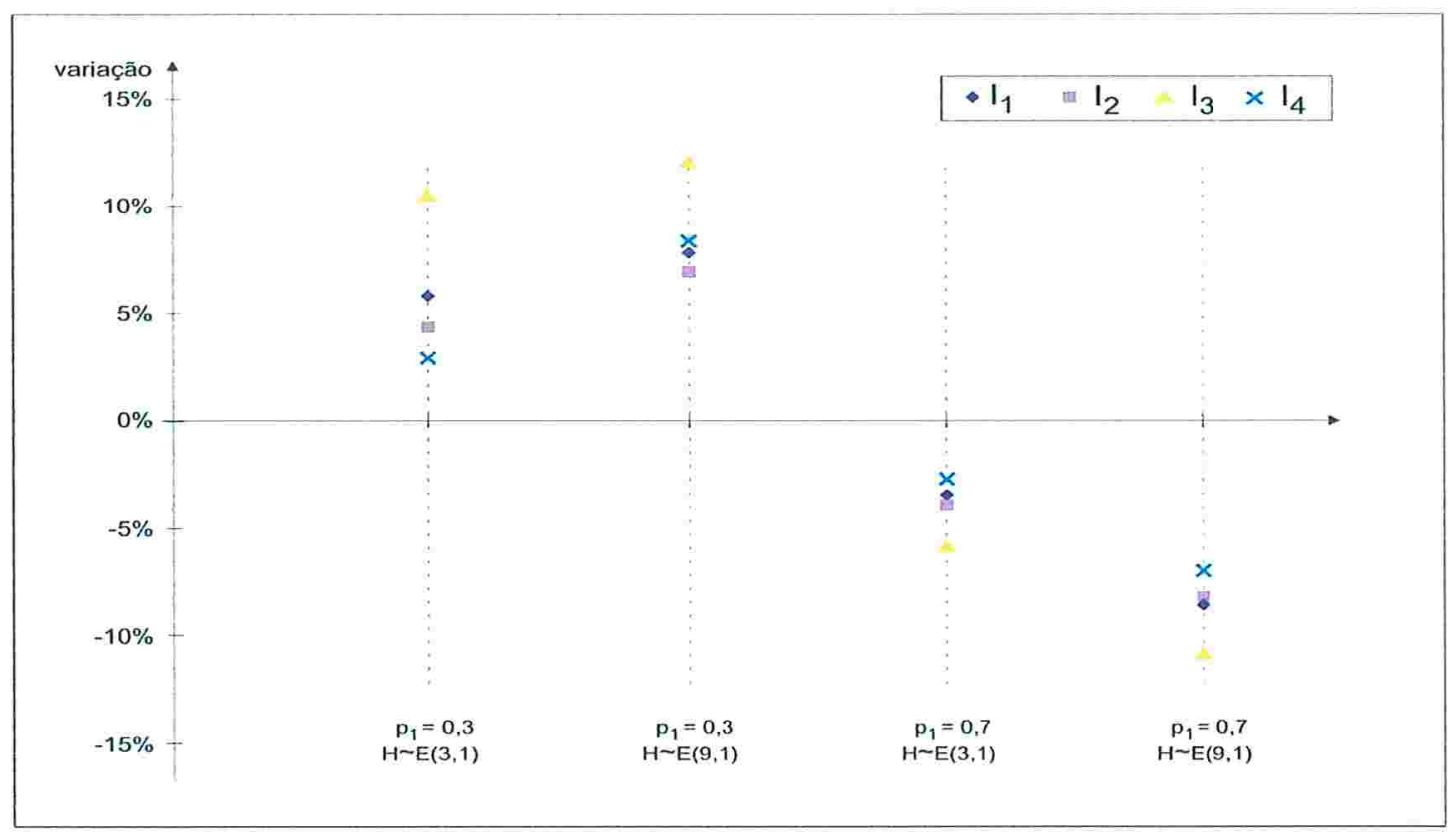

Figura 3.4: Variação no caso $k=200$ e serviços: $G_{1} \sim U(0,4)$ e $G_{2} \sim U(2,6)$.

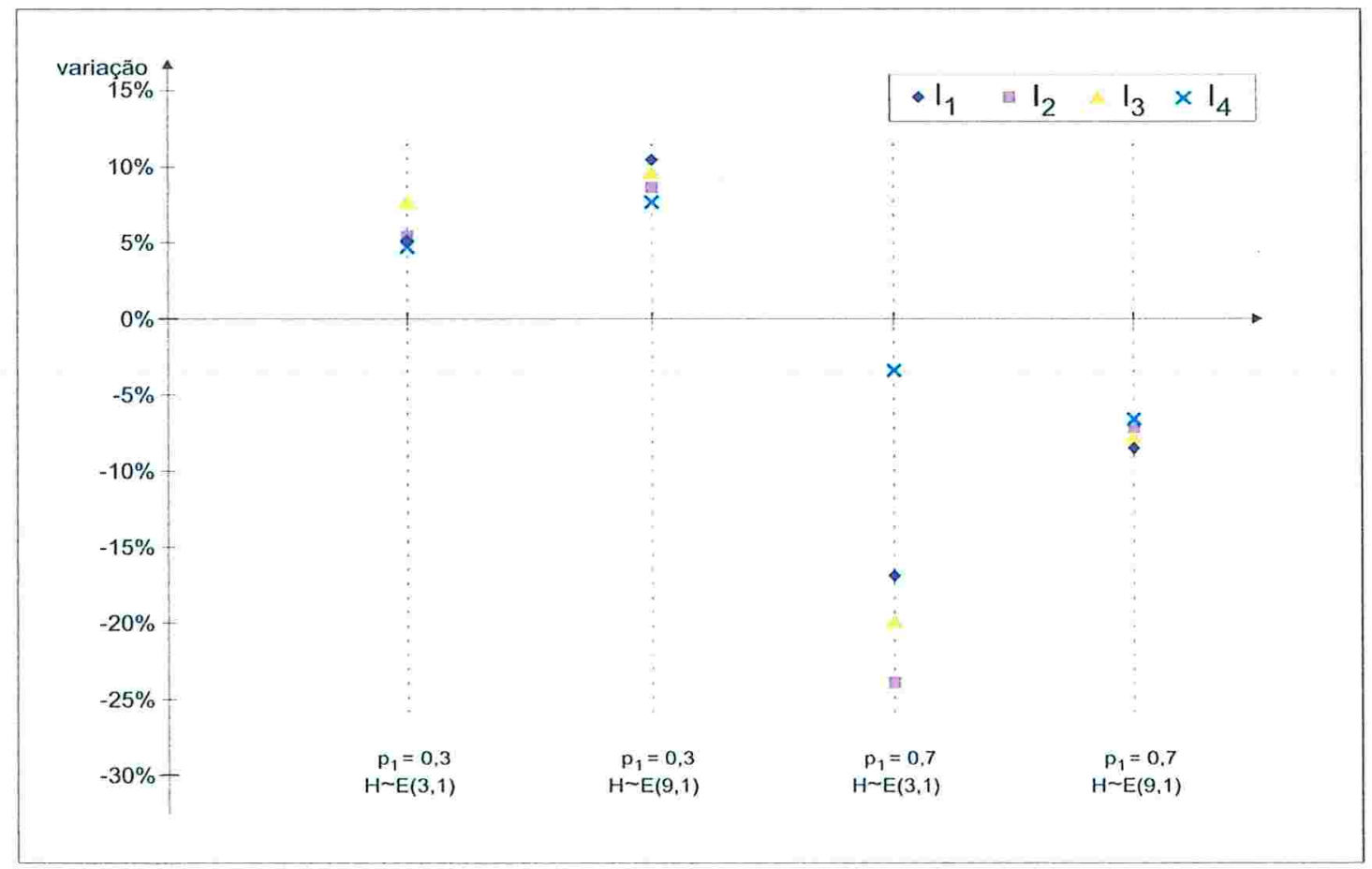

Figura 3.5: Variação no caso $k=200$ e serviços: $G_{1} \sim E(2,1)$ e $G_{2} \sim E(4,1)$. 


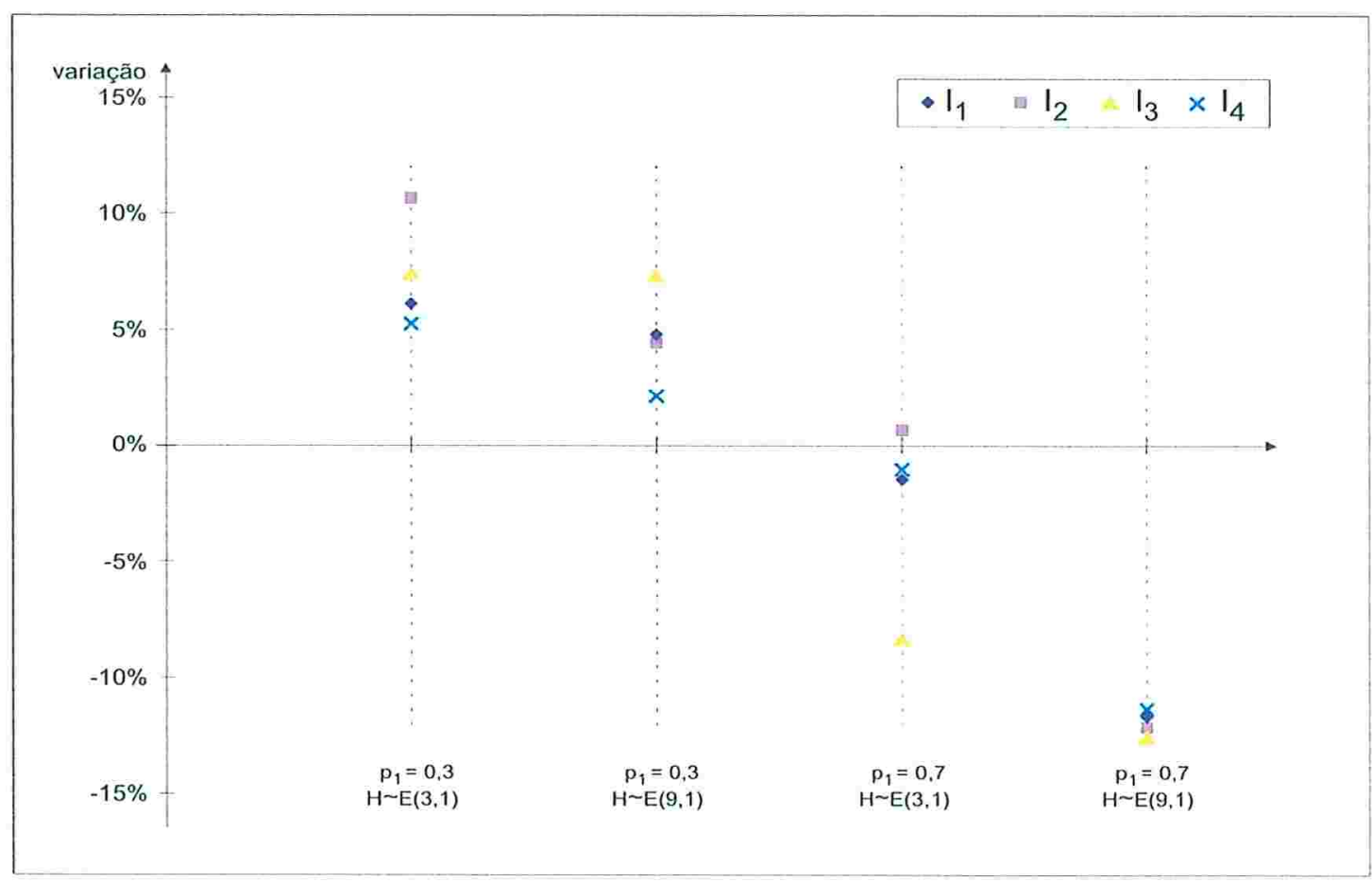

Figura 3.6: Variação no caso $k=200$ e serviços: $G_{1} \sim L N(2,1)$ e $G_{2} \sim L N(4,1)$.

muito próxima do obtido via simulação, o que merece ser estudado de forma mais detalhada cm trabalhos futuros. 


\subsubsection{Efeitos}

\section{Proporção de clientes do tipo 1}

Consultando as Tabelas (3.2) a (3.7), quando há predominância clientes com tempo de serviço mais breves, observamos que há uma tendência, nas posições $k=50,100,200$ em superestimar o valor real, para os tempos de serviço com distribuições Uniformes e Erlangs, independente se o cliente na fila é pouco paciente ou mais paciente. Por outro lado, quando a maioria dos clientes é do tipo que gasta mais tempo em serviço, ocorre uma subestimação da aproximação em relação ao valor real, ver Figuras 3.7 a 3.13. Condições iniciais diferentes parecem não influenciar nesta tendência.

\section{Posição}

Ao considerarmos o desvio padrão do tempo de espera na simulação, observamos que o valor da aproximação está contido no intervalo para as posições $k=1,5,10,15,50$ e 100.

Para $k=200$ há considerável varição do valor aproximado em relação ao valor simulado, independente da proporção de clientes do tipo 1 , distribuições de tempo de serviço, paciência. Neste caso, a condição inicial $I_{4} \sim U(0 ; 0,1)$ apresentou melhor desempenho independente das distribuições dos tempo de serviço. E a condição inicial $I_{3} \sim U(1,75 ; 2)$, piorou os resultados, pois poucas aproximações calculadas ficaram dentro do intervalo de confiança calculado.

\section{Paciência}

De modo sutil, a aproximação foi mais precisa, no caso em que havia clientes menos pacientes, $H \sim E(3,1)$, aguardando na fila e $k=200$.

\section{Condições iniciais}

A escolha da maior parte das condições iniciais utilizadas parecem não afetar o comportamento das tendências descritas anteriormente. A exceção, foi obtida, através 
da condição $I_{4}$, que representa um tempo já gasto em sistema ou fila, muito pequeno. 


\section{Capítulo 4}

\section{Conclusão}

\subsection{Resultados Obtidos}

Também modificamos a aproximação para o tempo médio de espera no modelo $M / G I / s / r+G I$ para incluir vários tipos de clientes ao alterarmos o instante em que é conhecido o tipo do cliente. Desenvolvemos um algorítmo para calcular numericamente o valor aproximado do tempo médio de espera e avaliamos as aproximações comparando-as com modelos de simulação. Consideramos dois tipos de clientes nos servidores e utilizamos distribuições de tempos de serviço Uniforme, Erlang e LogNormal com médias 2 e 4 para representar clientes que gastam, em média, pouco ou muito tempo para serem atendidos. Para os que aguardavam em fila, alteramos a distribuição da paciências entre distribuições Erlangs para representar clientes pouco ou muito pacientes.

De modo geral, a aproximação do tempo de espera em fila apresentada no Teorema 3, no Capítulo 3, parece ser razoável. O tempo médio de espera calculado pela expressão aproximada está contido no IC(95\%), quando o cliente encontra à sua frente menos que 100 clientes. Observamos que a aproximação obtida para quando há exatamente 100 clientes à sua frente parece ser boa, pois a condição inicial $I_{3}$, que teve menor porcentagem de valores contidos nos intervalos de confiança foi igual a $83 \%$. Já para o caso em que há mais que 200 clientes na fila, observamos que embora a condição inicial $I_{4}$, tenha apresentado 
uma boa porcentagem de valores inclusos no referido IC, as demais condições iniciais utilizadas apresentaram porcentagem de acerto, por volta dos $50 \%$. O que merece ser investigado em trabalho futuro com mais detalhes.

A escolha de diferentes condições iniciais parece não alterar de forma significativa a performance do cálculo do algoritmo, o que foi comprovado quando comparamos os valores da aproximação e da simulação para valores de tempos de serviço restante diferentes.

Vale ressaltar que observamos que quando há predominância clientes com tempo de serviço mais breves, observamos que há uma tendência, nas posições $k=50,100,200$ em superestimar o valor real, para os tempos de serviço com distribuições Uniformes e Erlangs, independente se o cliente na fila é pouco paciente ou mais paciente. Por outro lado, quando a maioria dos clientes é do tipo que gasta mais tempo em serviço, ocorre o contrário, uma subestimação da aproximação em relação ao valor real. Condições iniciais diferentes parecem não influenciar nesta tendência.

\subsection{Próximos passos}

Seria interessante efetuar o cálculo da aproximação para outras distribuições de tempo e serviço diferentes. Em particular, para os casos extremos, onde os tempos de serviço já gasto no sistema, podem ser todos muito pequenos ou muito grandes, quando os clientes estão sendo atendidos há pouco tempo ou quando estão prestes a receber o atendimento completo.

Além disso, pode ser interessante, verificar o comportamento da relação entre a aproximação e a simulação, para o caso em que há mais que dois tipos de clientes.

No caso da classificação de clientes, estes poderiam ser identificados no momento da chegada e não somente após o serviço ter sido iniciado.

Poderíamos também utilizar $H \sim M(n)$ para aproximar a distribuição da paciência, quando esta for uma distribuição GI. Além disso, poderíamos utilizar a aproximação $M(n) / M(n) / s / r+M(n)$ como sugerido no artigo [Brandt, 2002].

De modo geral, a pesquisa em Centrais de Atendimento tem várias frentes. Uma de- 
las é descrita a seguir. Um grande número de Centrais de Atendimento fornece o serviço mesclando atendimento realizado por agentes com o atendimento automatizado. Isto é, o serviço é feito através das unidades de resposta por voz (VRUs) e de agentes. Dentre os poucos artigos que modelam a utilização de VRU, está o de [Srinivasan, 2001] que analisa um modelo de redes com duas estações de VRUs atreladas, seguidas de serviço fornecido por agentes. Os detalhes desta interação ainda não foram suficientemente explorados na literatura especializada e se constitui em um trabalho desafiador de pesquisa. 


\section{Apêndice A}

\section{Algoritmo e Simulação Utilizando R}

\section{A.1 Algoritmo}

Distribuições de tempo de serviço Uniformes e tempo para abandonar Erlang $\mathrm{A}:=$ distribuicao do tempo entre chegadas;

(vetor com o nome da distribuicao no formato do $\mathrm{R}$, seguido com a lista de parametros) $\mathrm{G}:=$ distribuicoes do tempo de servico de acordo com a classe do cliente;

$\mathrm{s}:=$ quantidade de servidores;

$\mathrm{r}:=$ quantidade de lugares na central de atendimento;

$\mathrm{k}:=$ qtde de clientes em fila, quando os s servidores ja estao ocupados

$\mathrm{H}:=$ distribuicao do tempo que os clientes est $\tilde{A} £ o$ dispostos a esperar;

$\mathrm{m}:=$ quantidade de classes cle clientes;

p1 := probabilidade do cliente ser do tipo 1 ;

$\mathrm{p}:=$ probabilidade do cliente ser do tipo i com $1<=i<=m$;

$\mathrm{x}:=$ tempo de serviço ja gasto pelos clientes que estao ocupando os s servidores;

tipo $:=$ o tipo de cada cliente na posicao i nos s servidores no instante original;

$\mathrm{y}:=$ tempo de espera em fila para os $\mathrm{k}$ clientes no momento de uma nova chegada.

$\operatorname{rm}(\operatorname{list}=\operatorname{ls}())$

resultado $=\operatorname{list}()$ 


$$
\begin{aligned}
& \mathrm{s}=3 \\
& \mathrm{k}=4 \\
& \mathrm{p} 1=0.3 \\
& \mathrm{p}=\mathrm{c}(\mathrm{p} 1,1-\mathrm{p} 1) \\
& \mathrm{classes}=\mathrm{c}(1,2) \\
& \mathrm{a}=3 \\
& \mathrm{~s} 1=1 \\
& \mathrm{passo}=0.001 \\
& \text { digitos }=4 \\
& \text { Sorteio das classes } \\
& \text { tipo=rbinom(s,1,p)+1 }
\end{aligned}
$$

Gera valores de $\mathrm{x}$ válidos

$\mathrm{x}=$ NULL

for (i in $1: s)\{$

if $(\operatorname{tipo}[\mathrm{i}]==1) \times[\mathrm{i}]=\operatorname{round}(\operatorname{runif}(1,0,2)$, digitos $)$

else $x[i]=\operatorname{round}(\operatorname{runif}(1,0,2)$, digitos)

\}

Acumulada do tempo de serviço

$\mathrm{G}=$ function(tipo, $\mathrm{x})\{$

res $=$ NULL

for ( $\mathrm{j}$ in 1:length(tipo)) \{

if $(\operatorname{tipo}[\mathrm{j}]==1) \operatorname{res}[\mathrm{j}]=\operatorname{round}(\operatorname{punif}(\mathrm{x}[\mathrm{j}], \min =0, \max =4)$, digitos $)$

if $(\operatorname{tipo}[\mathrm{j}]==2) \operatorname{res}[\mathrm{j}]=\operatorname{round}(\operatorname{punif}(x[\mathrm{j}], \min =2, \max =6)$, digitos $)$

\}

return (res)

\}

Erlang Acumulada

$\mathrm{p} . \mathrm{Ek}=$ function $(\mathrm{x}, \mathrm{k}, \mathrm{s} 1)$ return $(\operatorname{round}(\operatorname{pgamma}(\mathrm{x}, \mathrm{shape}=\mathrm{k}, \mathrm{scale}=\mathrm{s} 1)$, digitos $))$

Erlang $\mathrm{p}$ 
$\mathrm{r} . \mathrm{Ek}=$ function(rep,k,s1) return (round(rgamma(rep,shape=k,scale=s1), digitos))

Gera Erlang de acordo com o tipo do cliente

$\mathrm{G} . \mathrm{Ek}=$ function $($ tipo, $\mathrm{x})\{$

result $=$ NULL

for $(\mathrm{j}$ in 1:length(tipo)) \{

if $(\operatorname{tipo}[\mathrm{j}]==1)\{\operatorname{result}[\mathrm{j}]=\operatorname{round}(\mathrm{p} \cdot \operatorname{Ek}(\mathrm{x}[\mathrm{j}], 2,1)$, digitos $)\}$

else $\{\operatorname{result}[\mathrm{j}]=\operatorname{round}(\mathrm{p} \cdot \operatorname{Ek}(\mathrm{x}[\mathrm{j}], 4,1), \operatorname{digitos})\}$

\}

result

\}

Erlang condicional

G.Ek.cond $=$ function $($ tipo,t,x)

$\operatorname{return}(((\mathrm{G} \cdot \operatorname{Ek}(\operatorname{tipo}, \mathrm{t}+\mathrm{x})$ - G.Ek(tipo,x $)) /(1$ - G.Ek(tipo,x $))))$

Acumulada da paciência

$\mathrm{H}=$ function( $\mathrm{x})$ return $(\mathrm{p} \cdot \operatorname{Ek}(\mathrm{x}, \mathrm{a}, \mathrm{s} 1))$

Condicionais $\mathrm{G}$ e $\mathrm{H}$

G.cond $=$ function (tipo, $\mathrm{t}, \mathrm{x}$ )

return $((\mathrm{G}($ tipo, $\mathrm{t}+\mathrm{x})-\mathrm{G}($ tipo, $\mathrm{x})) /(1-\mathrm{G}($ tipo, $\mathrm{x})))$

$H . c o n d=$ function $(t, y)$

return $((\mathrm{H}(\mathrm{t}+\mathrm{y})-\mathrm{H}(\mathrm{y})) /(1-\mathrm{H}(\mathrm{y})))$

Inicia o tempo de espera dos $\mathrm{k}$ clientes que estão em fila

$\mathrm{y}=\operatorname{round}(\operatorname{runif}(\mathrm{k}, \min =0, \max =2)$, digitos $)$

$\mathrm{z}=\mathrm{y}$

$\mathrm{y}=\operatorname{sort}(\mathrm{z}$, decreasing $=\mathrm{TRUE})$

Inicializa o valor do tempo

$\mathrm{t}=$ passo

servico.restante $=\operatorname{sum}($ G.cond $($ tipo, $t, x))$

Cálculo do $t_{1}$

Encontra o menor tempo para que somatória dos tempos restantes seja igual a 1 


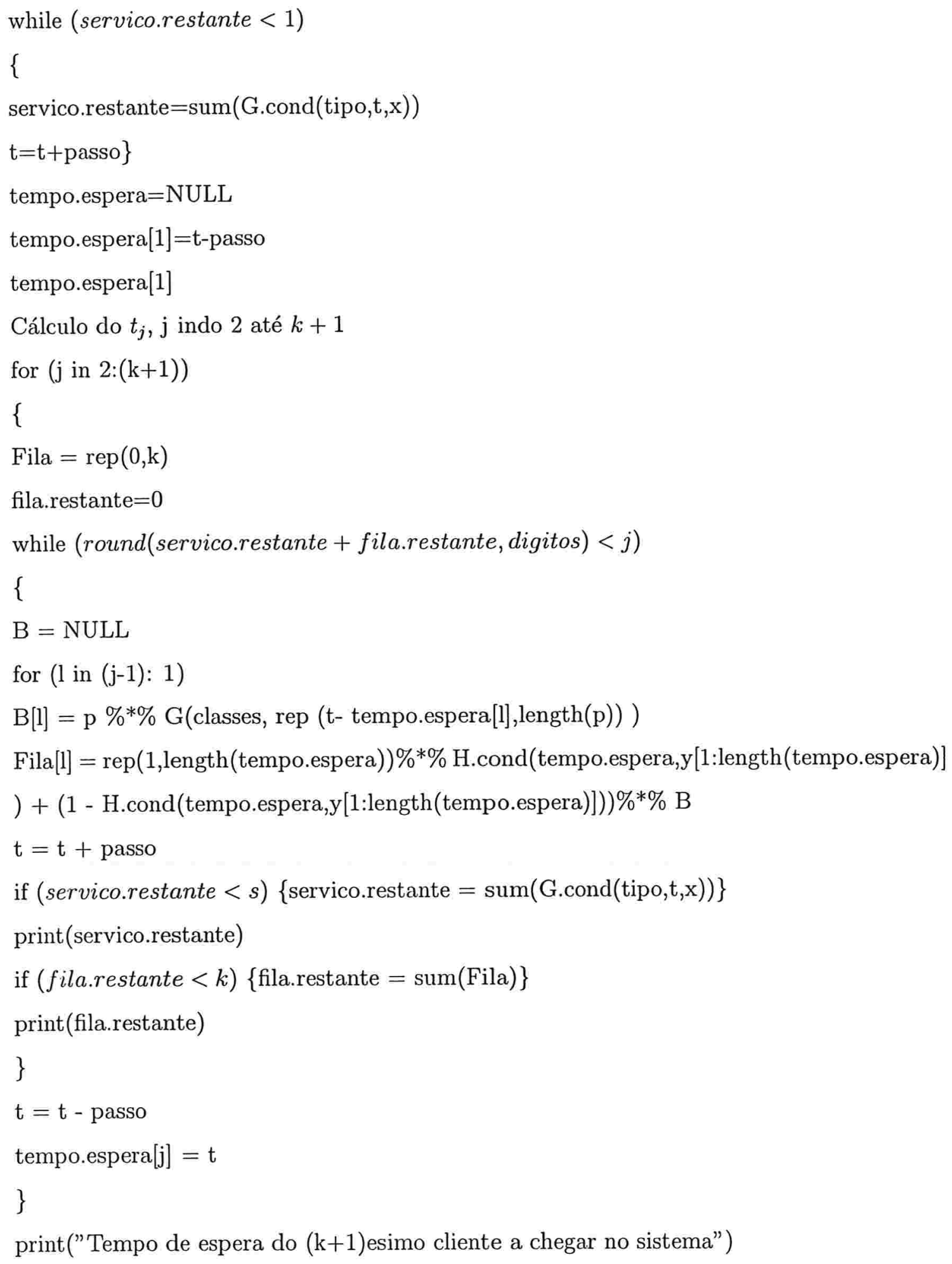


tempo.espera[ $[\mathrm{k}+1]$

\section{A.2 Simulação}

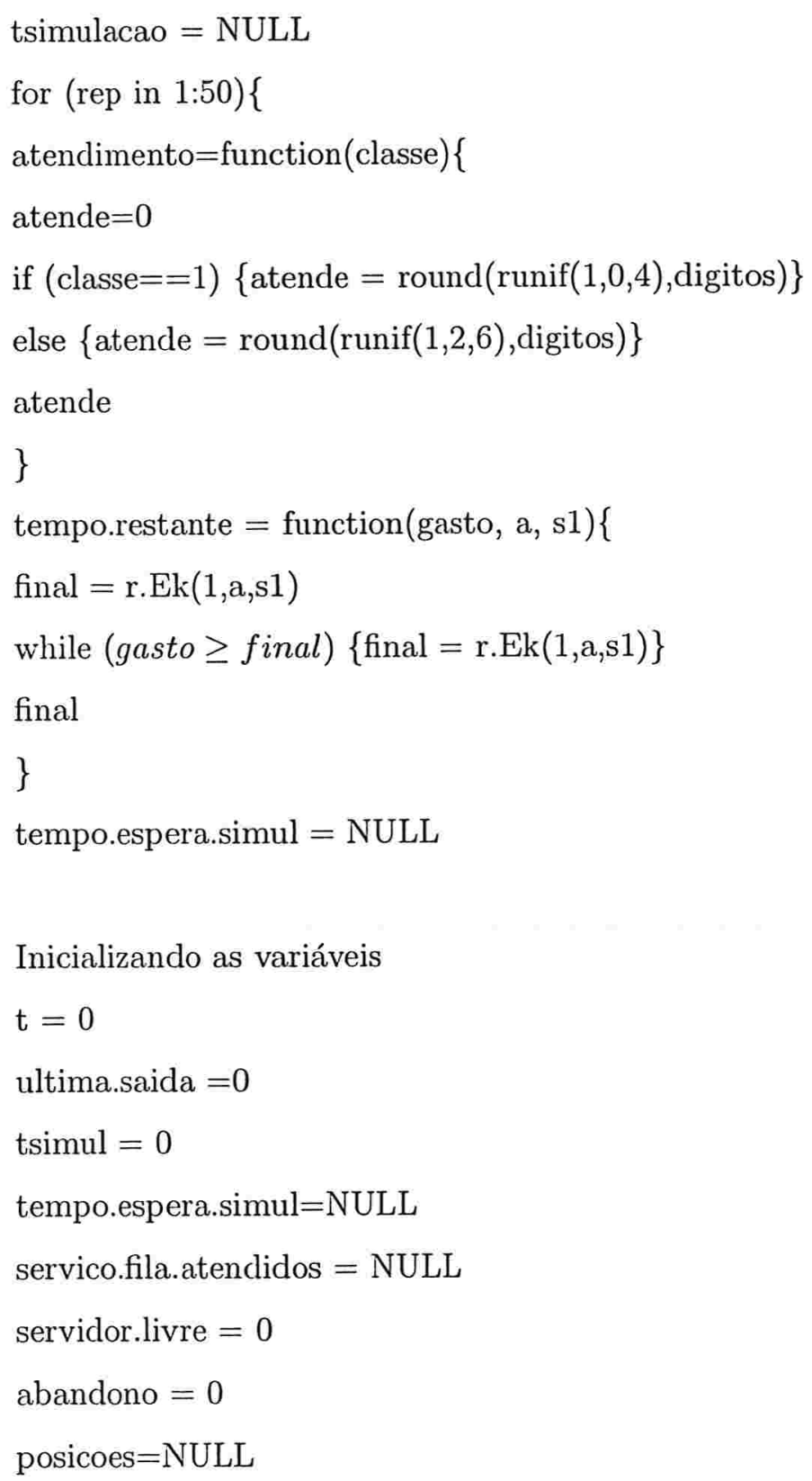


$j=1$

Sorteia o tempo total de serviço para os s clientes que estão sendo atendidos no momento da chegada do $(k+1)$-ésimo cliente

fim.servico $=$ NULL

for ( $\mathrm{i}$ in $1: \mathrm{s})\{$

if $(\operatorname{tipo}[\mathrm{i}]==1)$ fim.servico[i] $=\operatorname{round}(\operatorname{runif}(1, \max (0, x[\mathrm{i}]), 4), \operatorname{digitos})$

else fim.servico[i] $=\operatorname{round}(\operatorname{runif}(1, \max (2, x[i]), 6)$,digitos $)$

\}

fim.servico

fim.servico.aux $=$ fim.servico

Sorteia os tempos de paciencia

fim.paciencia $=\mathrm{y}$

for ( $\mathrm{i}$ in $1: \mathrm{k})\{$

fim.paciencia $[\mathrm{i}]=$ tempo.restante $(\mathrm{y}[\mathrm{i}], \mathrm{a}, \mathrm{s} 1)$

\}

fim.paciencia.aux =fim.paciencia

Sorteia a classe do cliente que está na fila

tipo.fila $=$ NULL

tipo.fila $=\operatorname{rbinom}(k, 1, p)+1$

tipo.fila

tipo.fila.aux=tipo.fila

$\mathrm{t}=\min ($ fim.servico $)$

tempo.espera.simul $[\mathrm{j}]=\mathrm{t}$

servidor.livre $=$ which.min $($ fim.servico $)$

while (length(tipo.fila) $>0)\{$

Verificando as desistencias, se houve

mais.impaciente $=\min ($ fim.paciencia $)$

if (mais.impaciente $>t$ ) \{ 


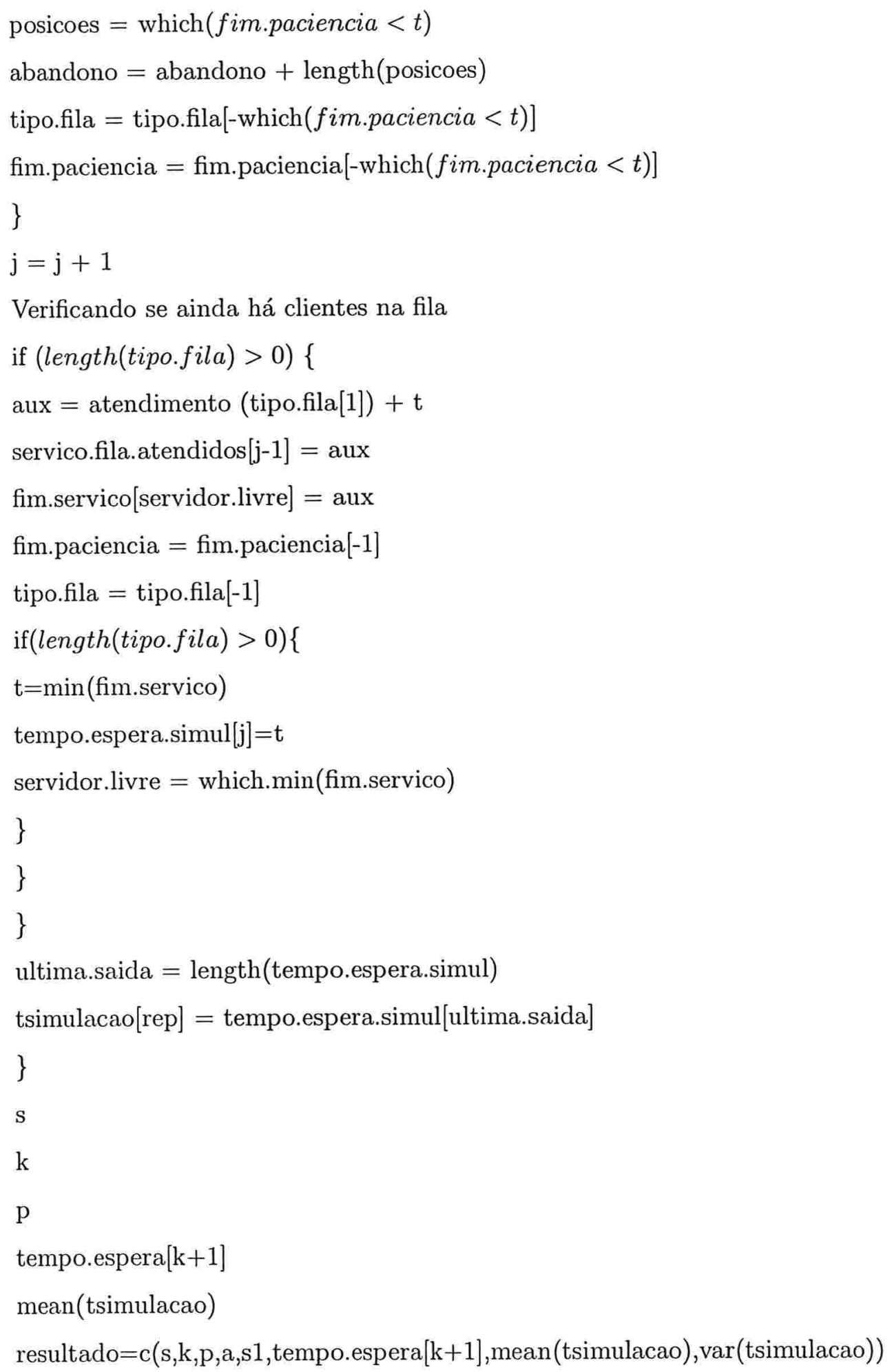




\section{Referências Bibliográficas}

Berger, A. \& Whitt, W. (1992). The impact of a job buffer in a token-bank rate controlthrottle, Stochastic Models 12: 215-243.

Brandt, A. \& Brandt, M. (2002). Asymptotic results and a markovian approximation for the $m(n) / m(n) / s+g i$ system, Queueing System 41: 73-94.

Feinberg, R., Kim, I., Hokama, K., de Ruyter, K. \& Keen, C. (2000). Operational determinants of caller satisfaction in the call center, International Journal of Service Industry Managment 11: 131-141.

Magalhães, M. N. (2006). Probabilidade e Variáveis Aleatórias, IME-USP, SÃO PAULO.

N., G., Koole, G. \& Mandelbaum, A. (2005). Telephone call center: Tutorial, review and research prospects, Manufacturing Service Operations Management 5: 79-141.

Srinivasan, R. \& Talimt, J. (2001). Performance analysis of a call center with interacting voice response units, Tecnical Report - University of Saskatchewan .

Whitt, W. (1984). Approximations for departure processes and queues in series, Naval Res. Logist. Quart. 31: 499-521.

Whitt, W. (1999a). Improving service by informing customers about anticipated delays, Management Science 45: 192-207.

Whitt, W. (1999b). Predicting queueing delays, Management Science 45(6): 870-888. 
Whitt, W. (2005). Engineering solution of a basic call center model, Management Science 51: 221-235. 